
\title{
2D boron nitride nanosheets for polymer composite materials
}

\author{
Md Golam Rasul $\mathbb{D}^{1}$, Alper Kiziltas ${ }^{2}$, Babak Arfaei $\mathbb{D}^{3 凶}$ and Reza Shahbazian-Yassar $\mathbb{D}^{1 凶}$
}

Hexagonal boron nitride nanosheets (BNNSs) are promising two-dimensional materials to boost the mechanical, thermal, electrical, and optical properties of polymer nanocomposites. Yet, BNNS-polymer composites face many challenges to meet the desired properties owing to agglomeration of BNNSs, incompatibility, and weak interactions of BNNSs with the host polymers. This work systematically reviews the fundamental parameters that control the molecular interactions of BNNSs with polymer matrices. The surface modification of BNNSs, as well as size, dispersion, and alignment of these nanosheets have a profound effect on polymer chain dynamics, mass barrier properties, and stress-transfer efficiency of the nanocomposites.

npj 2D Materials and Applications (2021)5:56; https://doi.org/10.1038/s41699-021-00231-2

\section{INTRODUCTION}

Boron nitride nanosheet (BNNS)-polymer composites are one of the important classes of materials with a wide range of applications spanning from automotive ${ }^{1-3}$, aerospace ${ }^{4,5}$, healthcare and medicine $e^{6-8}$, energy storage ${ }^{9-11}$ to electronic engineering ${ }^{12}$. While many efforts have been devoted to graphene incorporation into polymer materials ${ }^{13-19}$, BNNS-polymer composites are less explored. Boron nitride (BN) nanomaterials have superior fracture strength $(165 \mathrm{GPa})$, high Young's modulus $(0.8 \mathrm{TPa})^{20}$, high thermal stability (up to $800^{\circ} \mathrm{C}$ in the air) ${ }^{21,22}$, excellent thermal expansion coefficient $\left(-2.72 \times 10^{-6} \mathrm{~K}^{-1}\right)^{23}$, and outstanding thermal conductivity $\left(300-2000 \mathrm{Wm}^{-1} \mathrm{~K}^{-1}\right)^{22,24,25}$. Unlike other two-dimensional (2D) materials such as graphene and MXene ${ }^{26-30}$, BNNS is electrically insulative due to the wide bandgap $(\sim 5.6 \mathrm{eV})$ with a dielectric constant $(4 \sim 8)$, low leakage current, and high voltage breakdown strength $\left(12 \mathrm{MV} \mathrm{cm}^{-1}\right)^{31}$. These characters suggest that BNNS-polymer nanocomposites can be engineered with superior performances.

Although both BN and carbon nanotube (CNT) nanomaterials show van der Waals $(\mathrm{vdW})$ force and $\pi-\pi$ interactions with polymers, the partial ionic electronic structure of B-N bonding can be advantageous in terms of molecular interactions with polymer materials ${ }^{32-36}$. Molecular dynamics (MD) simulation predicted that the molecular interaction between polymers and BN materials could be more efficient when compared with carbon nanofillers because of the polarized nature of B-N bonding, the high bonding potentials (deep potential well) of $\mathrm{B}$ and $\mathrm{N}$ atoms, along with vdW force and Coulomb interactions between $\mathrm{BN}$ and polymer matrix $^{37,38}$. As a result, there would be better opportunities to enhance the molecular interactions between BN materials and polymers if nanocomposites are properly designed and synthesized. Consequently, the number of publications in the field of BNpolymer nanocomposites are increased significantly in recent years (Fig. 1).

The focus of this review article is to investigate the factors that govern the incorporation of BN nanosheets (BNNSs) into polymer materials. In particular, the interfacial and non-interfacial engineering between BNNSs and polymers are critically discussed to address the low stress-transfer, BNNS agglomeration, and failure mechanisms in polymer composites. Here, we outline the important material design factors that govern mechanical reinforcement (tensile modulus and strength), as well as the thermal (thermal conductivity, glass transition temperature $\left(T_{\mathrm{g}}\right)$, and thermal stability), electrical (dielectric breakdown strength, electrical resistivity, and $\mathrm{Li}^{+}$ion conductivity), and optical (optical transparency, IR absorption, ultraviolet (UV) absorption, and filtering) properties of BNNS-polymer nanocomposites. Notably, the key findings of our study can also be extended for other 2D materials such as graphene and MXene composite systems. This review is quite distinct from other published review papers on $\mathrm{BN}$ polymer composite materials ${ }^{24,25,39-44}$. Zhi et al. ${ }^{42}$, Zheng et al. ${ }^{44}$, and Weng et al. ${ }^{25}$ reviewed the functionalization of boron nitride nanotubes (BNNTs) and BNNSs to enhance the processability and solubility for versatile applications of their nanocomposites. Verma and co-workers ${ }^{41}$ demonstrated different atomistic modeling techniques for engineering polymer-BN composites. Wang et al. ${ }^{39}$, Yu et al. ${ }^{40}$, and Meng et al. ${ }^{24}$ focused primarily on thermal conductivity of BN-polymer nanocomposites. However, these review articles fall short in determining the important underlying factors that govern the mechanical reinforcement, thermal, electrical, and optical property changes in BNNS-polymer nanocomposites. Moreover, $T_{\mathrm{g}}$, thermal stability, and optical properties of BNNS-incorporated polymer nanocomposites were not reviewed before. We also have outlined emerging applications of the BNNS-polymer composites that require addressing the critical challenges of such composite systems.

\section{STRUCTURE AND PROPERTIES OF BORON NITRIDE}

Hexagonal boron nitride (hBN) is the most stable form under standard conditions among all three crystalline structures (hBN, cubic $\mathrm{BN}$, and wurtzite $\mathrm{BN}$ ). $\mathrm{hBN}$ is available in zero-dimensional (OD fulborenes), one-dimensional nanotube (1D BNNT), and 2D nanosheet (2D BNNS) forms. In the atomic structure of hBN, B and $\mathrm{N}$ atoms are bonded with a strong covalent bond in a honeycomb configuration similar to graphene. Multiple BN layers are attached with weak vdW forces. They are oriented in such a way that B and $\mathrm{N}$ atoms are placed above and below each other in adjacent layers $^{25,45}$.

Key properties, including crystal structure parameters of bulk BN, few-layer BNNSs, BNNT, and graphene nanomaterials, are demonstrated in Table 1. The Young's modulus, fracture strength, and crystallographic structure information of BN materials are similar to graphene. However, BN has better thermal stability and

\footnotetext{
${ }^{1}$ Mechanical and Industrial Engineering Department, University of Illinois at Chicago, Chicago, IL, USA. ${ }^{2}$ Research and Innovation Center, Ford Motor Company, Dearborn, MI, USA

${ }^{3}$ Research and Advanced Engineering, Ford Motor Company, Palo Alto, CA, USA. ${ }^{凶}$ email: barfaei@ford.com; rsyassar@uic.edu
} 


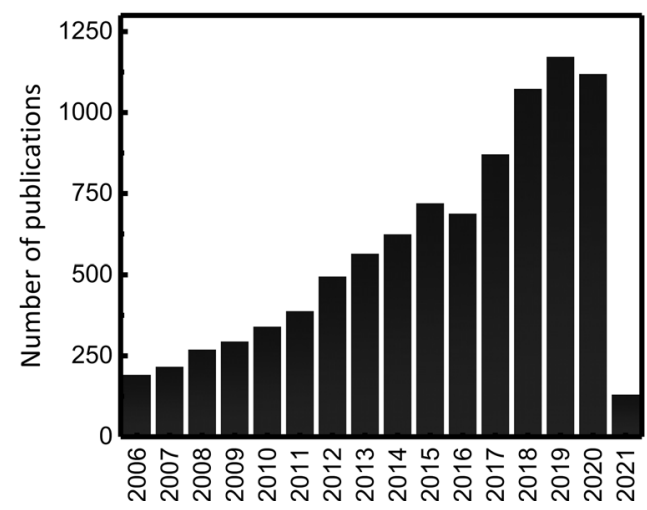

Fig. 1 Publication number of polymer-BN composites. Number of BN-based polymer composite publications from 2006 to 2021. (Keywords used for searching "polymer" and "boron nitride". Source: www.scifinder.cas.org, accessed in February 2021).

a significantly higher electronic bandgap compared with graphene, thus making it suitable for electrical insulation application. Although most of the properties are comparable for all three types of BN nanomaterials (bulk BN, few-layer BNNS, BNNT), few-layer BNNSs have very high surface area $\left(\sim 2600 \mathrm{~m}^{2} \mathrm{~g}^{-1}\right)$ compared to bulk BN $\left(\sim 10 \mathrm{~m}^{2} \mathrm{~g}^{-1}\right)$ and BNNT $\left(212-254 \mathrm{~m}^{2} \mathrm{~g}^{-1}\right)$. The thermal conductivity of mono/few-layer BNNSs show significantly higher values ( $2000 \mathrm{~W} \mathrm{~m}^{-1} \mathrm{~K}^{-1}$ ) compared with bulk BN materials ( $390 \mathrm{~W}$ $\mathrm{m}^{-1} \mathrm{~K}^{-1}$ ). In 2D morphology, BNNSs have a high aspect ratio that facilitates high surface area for interfacial molecular interaction within nanocomposite matrix ${ }^{24}$. Thus, a maximum amount of reinforcement can be realized into a polymer matrix using a very low amount of BNNSs.

\section{FACTORS AFFECTING BNNS-POLYMER NANOCOMPOSITE MATERIALS}

The nanocomposite properties depend on a wide range of factors, including molecular interactions between BNNSs and polymers, BNNSs surface area, interphase properties, interface spacings or voids formed at the polymer-BNNSs interfaces, dispersion of BNNSs within nanocomposite matrix, and processing parameters. These elements are categorized into two broad thrusts: (i) interfacial and (ii) non-interfacial factors (Fig. 2) in this review for systematic analysis of the corresponding nanocomposites. The following sections (Sections 3.1-3.5) provide an overview of these factors, and later these factors are discussed for polymer-BNNS composites properties such as mechanical (Section 4.1), thermal (Section 4.2), electrical (Section 4.3), and optical (Section 4.4).

\section{Physical and chemical interactions between BNNSs and polymers}

Interfacial interaction is one of the fundamental part of understanding the corresponding polymer nanocomposite materials because they govern the mechanical, thermal, electrical, and optical properties of the materials ${ }^{46}$. Poor interfacial interactions between BNNSs and polymer matrix may result in BNNSs slippage, ineffective load transfer, and void formation, resulting in poor nanocomposite properties. Interfacial interactions could be divided into two types: physical and chemical. Physical interactions involve morphological and structural activations of BNNSs, which are very weak; consequently, the interphase strength is also weak $^{47-49}$. On the other hand, chemical interactions including covalent and non-covalent interactions are stronger than physical ones ${ }^{47-49}$. Although BN shows a partially ionic character, the inertness of BN still makes it challenging to form favorable interactions with polymers. Such limitations could be overcome by

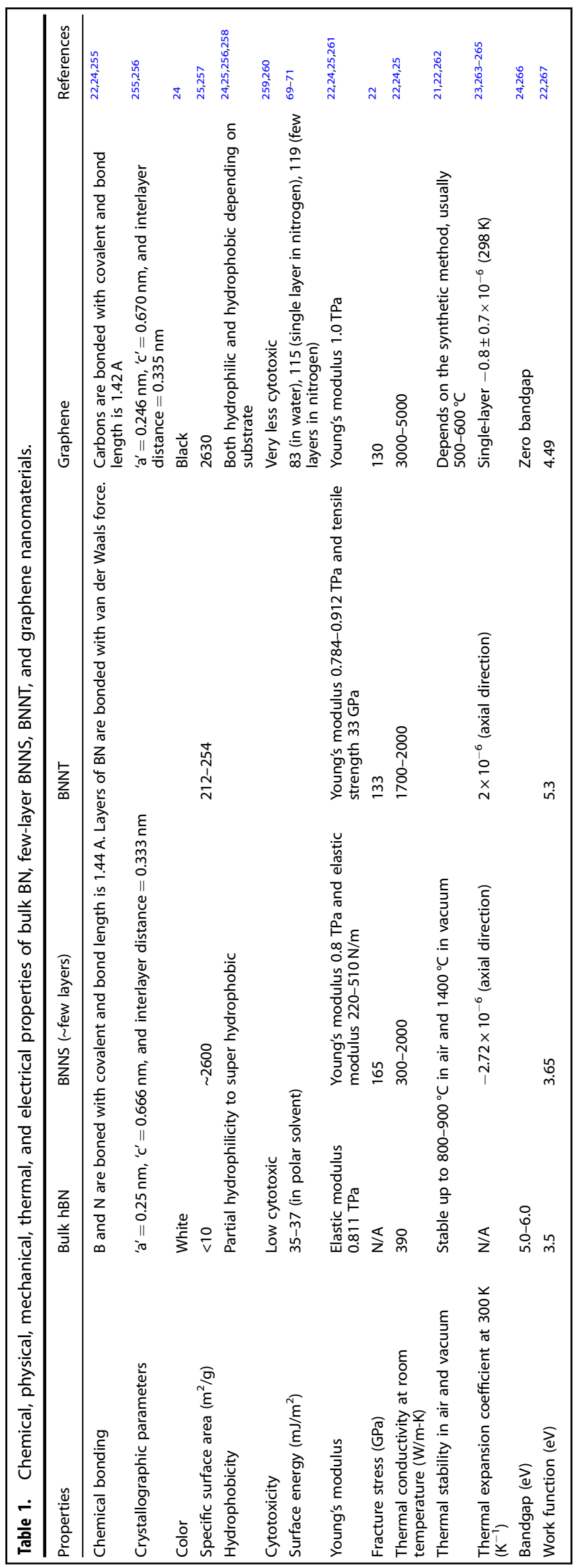




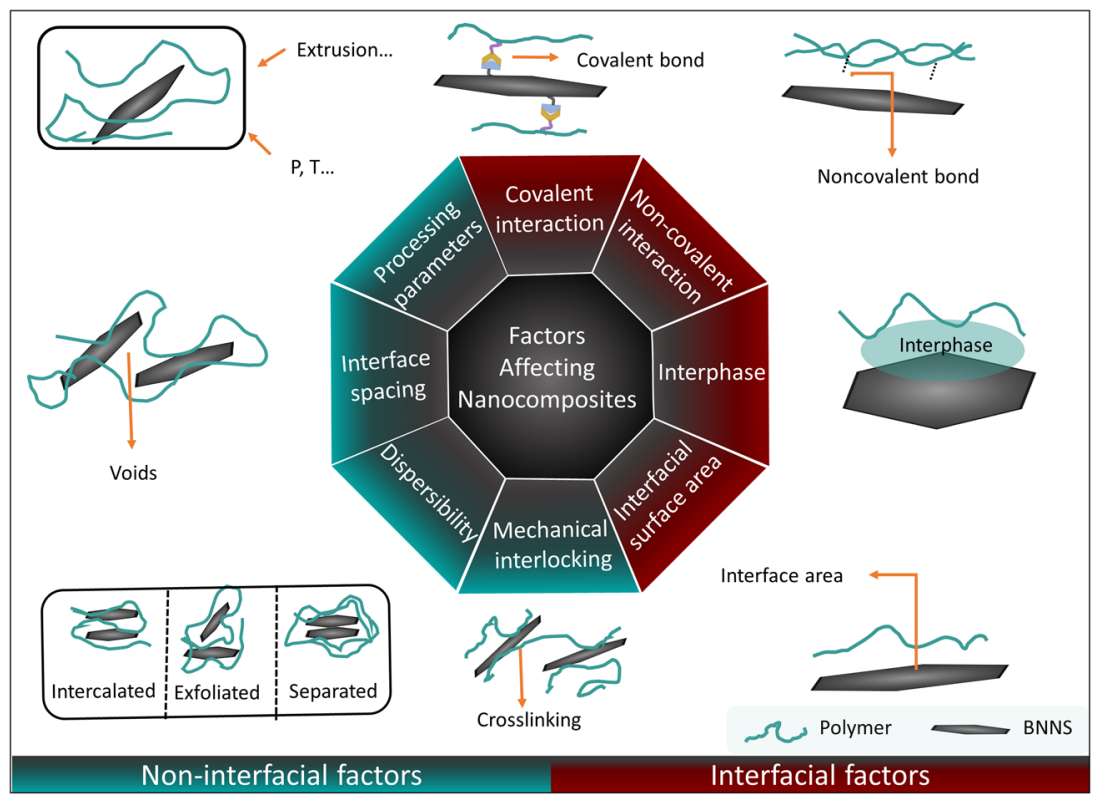

Fig. 2 Factors affecting BNNS-based polymer nanocomposite properties. Interfacial factors include covalent and non-covalent interactions, interfacial surface area, and interphase at the BNNS-polymer interface. Non-interfacial factors include dispersibility, mechanical interlocking, interface spacing, and processing parameters. These factors modify polymer nanocomposite properties by engineering chain dynamics, load or stress transfer, and mass barrier.

functionalizing BNNSs with appropriate functional groups. As a result, molecular interactions between BNNSs and polymers could be ameliorated, resulting in: (i) increased compatibility by reducing the surface energy difference between polymers and BNNSs and (ii) uniform dispersion of BNNSs into organic/inorganic solvent/matrix ${ }^{50}$.

A summary of chemical functionalization (covalent and noncovalent) of BNNSs using different types of molecules is demonstrated in Fig. 3. In spite of having enhanced bonding strength, covalent functionalization might not be suitable for some particular applications such as electrical and optical owing to the structural damages caused by functionalization ${ }^{51}$. Such functionalization could modify the electronic band structure via introduction of impurities/defects in the BN crystal structure. However, these impurities/defects-induced structurally damaged $2 \mathrm{D}$ materials are likely to either decrease or increase the thermal conductivity of the composite materials depending on the type of defects $^{52}$. To overcome such limitations, non-covalent functionalization with molecules as such aniline, amine, and acidic groups are also shown in Fig. 3. Non-covalent functionalization involves creating $\mathrm{H}$-bond, vdW force, Coulomb interactions, and $\pi-\pi$ stacking of BNNSs with functional molecules. Functional groups such as hydroxyl, acidic, and amine are suitable to form $\mathrm{H}$-bond with hydrophilic polymer matrices ${ }^{25,53}$. In contrast, silane groups are compatible with both hydrophilic and hydrophobic types of polymer matrices ${ }^{54,55}$. BNNSs functionalization with appropriate molecules is, therefore, necessary to design and construct the interfacial layers between BNNSs and polymer resins.

\section{BNNS-polymer interfacial surface area and interphase}

Molecular interactions at the BNNS-polymer interface create a transitional zone, termed as interphase, which is the origin of property changes in polymer nanocomposites ${ }^{56}$. Interphases have a vital role in transferring mechanical stress, thermal heat, and electrical load from one phase into another ${ }^{57-63}$. Several interphase modeling works were developed, where increase in modulus and strength was explained by polymer chain adsorption $^{64}$, as well as deformational interphase formation around BNNSs $^{65}$. By studying microstructural and micromechanical properties of nanocomposites, the interfacial adhesion strength could be tailored by the surface chemistry of nanofillers and polymer functionalities ${ }^{64}$. Kovalev et al. ${ }^{65}$ proposed a deformational interphase model where transition zone was accountable for variable gradient layers. This graded approach offered the transparent consideration of the gradient of the mechanical properties and introduced the transfer of the mechanical deformation between layers represented by a specifically selected transfer function. In addition, high surface area to volume ratio of BNNSs facilitates in enhancing molecular interaction with polymer matrix, thus creating higher interfacial volume in a composites than that of composites formulated from lower surface area nanosheets at the same loading amount ${ }^{66}$. Such high surface area to volume ratios could be achieved by downsizing the micro-size BN nanomaterials into nanosized particles or sheets. Another method used to synthesize high specific surface area BNNSs (i.e., $140 \mathrm{~m}^{2} \mathrm{~g}^{-1}$ ) was "chemical blowing technique", as reported in literature 67,68 . Together with high surface area to volume ratios, strong interphases at the polymer-BNNS interfaces will improve load transfer efficiency in polymer nanocomposites.

\section{Dispersibility and interface spacing}

Owing to the nanosheet structure and partially ionic configuration of BNNSs, surface energy of BNNSs $\left(35-37 \mathrm{~mJ} \mathrm{~m}^{-2}\right)$, vdW and electrostatic force among BNNSs are high ${ }^{69-71}$. These lead to agglomeration of BNNSs into most solvents and polymer matrices ${ }^{72-74}$. Agglomeration has significant detrimental effects on nanocomposite properties, including $T_{\mathrm{g}}$ from loosened chain dynamics ${ }^{75}$, reduced thermal conductivity from phonon scattering $^{76,77}$, low mechanical reinforcement from weak interphases ${ }^{78-80}$, and reduced optical transparency from light absorption ${ }^{81}$. Accumulation of BNNSs not only results in interphase volume reduction but also impede the percolating networks formation ${ }^{75}$. Defects, originated from the loss of interphase volume, can act as a phonon scattering. Thus, thermal conductivity of the polymer nanocomposites is reduced significantly ${ }^{76,77}$. In addition, tensile properties of nanocomposites suffer significantly owing to the loss of specific surface area and effective volume fraction of nanoparticles $^{79}$. Figure 2 shows three different type of BNNSs 


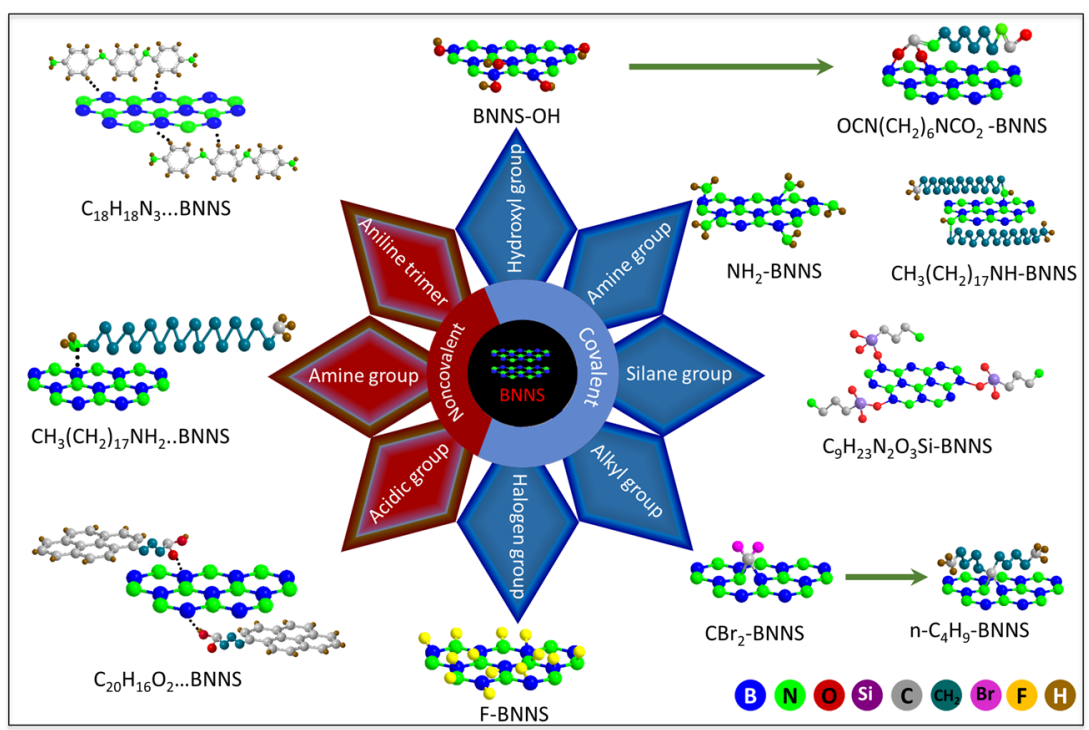

Fig. 3 Chemical functionalization of BNNSs. Covalent functionalization of BNNSs with hydroxyl group, amine group, silane group, alkyl group, and halogen group ${ }^{51,122,229-238}$. Non-covalent functionalization of BNNSs with acidic group, amine group, and aniline trimmer ${ }^{49,239-244}$. [Green arrows indicate conversion reaction to form other funcitonalized BNNS].

dispersion, which are usually found in nanocomposite matrices: intercalated, exfoliated, and separated or segregated. Exfoliated and intercalated BNNSs utilize maximum surface area per unit volume and are dispersed uniformly within nanocomposites compared with separated or segregated BNNSs. Techniques such as tuning surface energy, functionalizing BNNSs, and utilizing surfactants are utilized to overcome agglomeration within nanocomposites ${ }^{82,83}$. Moreover, nanocomposite processing such as twin-screw extrusion, stirring, and sonication are few other techniques that are developed to mitigate the agglomeration of BNNSs into nanocomposites ${ }^{84}$.

\section{Mechanical interlocking}

Polymer chain dynamics are influenced to a great extent owing to the mechanical interlocking effects of 2D nanofillers ${ }^{56,85-87}$. One of the widely used theories for explaining the mechanical interlocking effect into nanocomposites is "reptation doctrine" ${ }^{\text {"88 }}$. According to the reptation doctrine, polymer chains are confined in a transiently existing virtual tube, which is further surrounded by other polymer chains. If a force is applied on a polymer matrix, these chain entanglements create restrictive forces and are reflected in mechanical reinforcement. BNNSs act as a mechanical interlocking point and restrict polymer chain mobility owing to their spatial confinement. Thus, material properties that require chain movement, i.e., tensile modulus, strength, and $T_{\mathrm{g}}$ could be modified accordingly ${ }^{89}$. Peña-Bahamonde et al. ${ }^{89}$ reported an increase in $T_{\mathrm{g}}$ of polymer materials because of such mechanical interlocking effect that is originated from 2D nanofillers within nanocomposite matrix.

\section{Processing parameters}

Despite the fact that the wide range of nanocomposite fabrication methods are available today, BNNS-based polymer nanocomposites are mostly developed in three main approaches: in situ polymerization, melt-extrusion, and solution-blending ${ }^{90}$. In situ polymerization involves synthesis of polymer materials from monomers while solution-blending and melt-extrusion method involve compounding materials with the help of solvent and heat, respectively ${ }^{90}$. Such fabrication processes involve varieties of parameters as such mixing temperature and pressure for meltextrusion, solvent selection (polarity/non-polarity) and removal for solution-blending, reaction pressure, temperature, and chemical reactivity of the constituents for in situ polymerization ${ }^{90}$. These process conditions/parameters affect defect formation, agglomeration, and dispersion within nanocomposites, thus affecting the final material properties. On top of the commonly used fabrication methods, especially designed techniques such as ball milling, sonication, pressing, chemical modifications, chemical grafting, latex emulsion blending, layer-by-layer assembly, and direct assembly methods are also developed to incorporate nanofillers into polymer matrix ${ }^{91-100}$. Moreover, pre-, and post-processing techniques such as BNNSs alignment by magnetic field force or stretching are reported to improve anisotropic properties such as thermal conductivity into nanocomposites. For example, BNNSs alignment was demonstrated by Lin et al. ${ }^{101}$ using magnetic field and Jan et al. ${ }^{102}$ using material drawing operation. Such techniques are crucial to align BNNSs within nanocomposite matrix.

\section{BNNS-POLYMER NANOCOMPOSITE PROPERTIES Mechanical properties}

Ashby plot (Fig. 4) shows the elastic modulus and tensile strength of conventional elastomers, polymers, polymer-BNNS composites, and BNNSs materials. The modulus and strength of polymer-BNNS composites are scattered between polymers and BNNS materials, indicating the potential to develop super-strong materials with proper interfacial engineering.

The main factors affecting the reinforcement of BNNS-polymer nanocomposites include BNNSs aspect ratio (length to thickness ratio), BNNSs dispersion, efficient stress-transfer between BNNSs and polymer, and BNNSs alignment within the nanocomposite matrix. The stress-transfer between BNNSs and polymer materials is one of the most important parameters that allow transferring and distribution of external applied load disproportionately ${ }^{103}$. Stress induced within the nanofillers is proportional to the stress developed at the interphase ${ }^{104}$. Hence, the reinforcement effect depends mainly on the properties of interphase. The interphase fails at a critical external load, which is called interfacial fracture shear stress (IFSS). Figure 5 shows an illustration of the load/stress transfer at the interphase regions, where stresses or loads are propagating from one phase (polymer) to another (BNNS). Hence, 


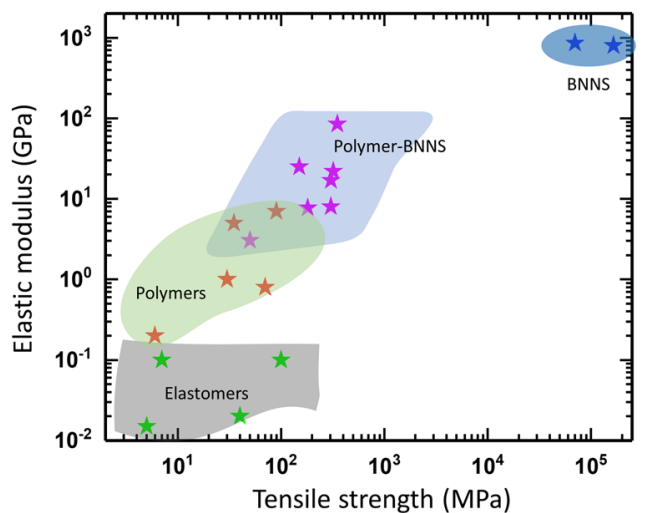

Fig. 4 Mechanical properties of pure polymers, polymer composites, and BNNS. Ashby plot of elastic modulus and tensile strength comparing the mechanical properties of conventional elastomers, polymers, polymer-BNNS composites, and BNNSs. Different color regions represent families of each material. The mechanical properties of polymer-BNNS composites are scattered between polymers and BNNSs in this Ashby plot, indicating to develop super-strong polymer composite materials in the near future if properly designed and manufactured. Ashby plot was made use of reported values from many literatures cited in this review article. References: Ashby, M. F. Material and Process Selection Charts, Chart created using CES EduPack 2018, Granta Design Ltd, and literature reports from refs. $49,67,81,92,102,105,108-110,112,113,132,140,152,156,183,233,236,245-249$.

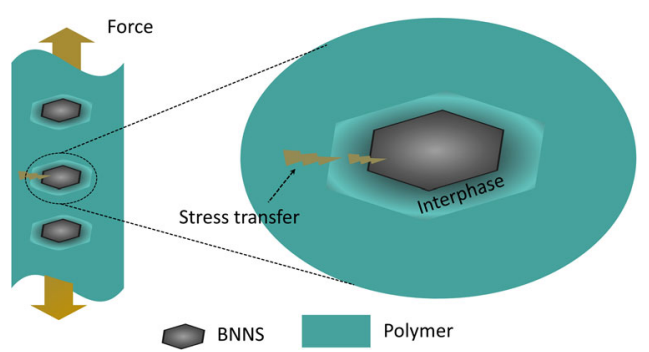

Fig. 5 Mechanical load/stress transfer in polymer composites. A schematic illustration of the load/stress transfer at the interphase regions where stresses or loads are propagating from one phase to another ${ }^{250-252}$. The increased surface area of BNNS and interphase strength facilitate to increase effective load/stress transfer from the polymer matrix to BNNSs.

it is crucial to improve the intrinsic properties of interphase by maximizing the volume of the interfacial regions and strengthening by forming chemical bonds among constituents. Uniform dispersion and increased aspect ratios or surface area to volume ratio of BNNSs are desirable to maximize the volume of the interfacial region. At the same time, physical and chemical bonding between fillers and matrix could facilitate to developing stronger interphases.

In order to maximize the load transfer at the interphase regions, the aspect ratios or surface area to volume ratio of nanosheets need to be increased since a high surface area of BNNSs could interact more with polymer chains compared with lower surface area BNNSs at same amount of loading. Such result was illustrated by assessing mechanical reinforcement for poly [2,2-(p-oxydiphenylene)-5,5'-bibenzimidazole]-BN composite systems where exfoliated and non-exfoliated BN nanomaterials were utilized ${ }^{105}$. The tensile modulus was increased by $3 \%(3.3-3.4 \mathrm{GPa})$ for nonexfoliated BN nanomaterials as opposed to $18 \%$ for exfoliated BNNSs at the same 4 wt.\% loading level. Exfoliation increases aspect ratios of BNNSs, thus creating a larger volume of interphase around BNNSs. As a result, increased load transfer could be achieved at the interface regions.

Such reinforcement of polymer composites is investigated by both simulation and experimental techniques, as such for polyethylene (PE)-BNNS composite systems ${ }^{106,107}$. While the experimental results reported an increase in the tensile strength and Young's modulus by 27 and $64.1 \%$, respectively, the MD simulation predicted the increment of the fracture stress by $21 \%$ at 5 wt.\% BNNS loading, indicating a good agreement with experimental results ${ }^{107}$. This paper also predicted that the aspect ratio, weight fraction, and geometry distribution of BNNSs (dispersed or stacked) significantly affected the mechanical properties of the composite materials ${ }^{107}$. The MD simulation carried out by Rouhi et al. ${ }^{38}$ found that the high surface area to volume ratio of BNNSs exposed more atoms, resulting in increased molecular interactions between polymer and BNNSs.

Although it is understandable to increase the interfacial surface area by loading more BNNSs, such high amount of BNNSs may lead to agglomeration because of the electronic structure of BNNS. The partial ionic electronic and 2D structure of BNNSs create electrostatic and vdW force interactions between BN nanosheets. With increasing the amount of BNNS loading, the net interaction energy becomes negative, resulting in agglomeration of nanosheets as suggested by the Derjaguin-Landau-VerweyOverbreak theory $^{74}$. Agglomeration is a major issue because the effective surface area of BNNSs can be significantly reduced, and agglomerated BNNSs may act as stress concentrating points making polymers to be vulnerable to early failure under tensile loading. As for example, polydimethylsiloxane (PDMS) polymers reported formation of BNNSs agglomeration within PDMS nanocomposites (Fig. 6) ${ }^{108}$. Boron nitride foams (BNFs) were added into the pure PDMS matrix to enhance the tensile strength, where BNFs could bear and resist the applied deformational force, thus increasing the tensile strength of the materials. The reinforcement of tensile strength was further increased from 1.65 to $1.95 \mathrm{MPa}$ by adding up to $8 \mathrm{wt} \%$ BNNSs. Interestingly, tensile strength was decreased from 1.95 to $1.7 \mathrm{MPa}$ when the amount of added BNNSs was further increased from 8 to $10 \mathrm{wt} . \%$. As evidenced by the SEM images in Fig. 6h, higher amount of BNNSs results in agglomerations, acting as a stress concentrating point and expedites crack propagation. Another study showed that 40 wt.\% BNNSs incorporated epoxy composites resulted in $\sim 80 \%$ tensile strength and $\sim 20 \%$ elastic modulus reductions ${ }^{109}$. High amount of BNNSs was likely accumulated within nanocomposites that failed earlier under tensile load than pure epoxy matrix. Besides acting as stress concentrating points, the accumulated BNNSs are suspected of creating heterogeneous microstructures within the nanocomposites and form defects ${ }^{110}$. Such heterogenous nanocomposite structures with defects are also vulnerable to early material failures under tension forces.

To avoid the accumulation of the BNNSs, several techniques have been developed, including functionalization, where appropriate organic/inorganic molecules are attached to the BNNSs. Polar functional molecules, i.e., amine $\left(\mathrm{NH}_{2}\right)$, hydroxyl $(\mathrm{OH})$, and silane, can be attached with BNNSs to increase the compatibility with polar polymers (e.g., nylon 6, epoxy, and poly(methyl methacrylate) (PMMA)) ${ }^{111}$. Similarly, second-degree alkyl amide and aniline trimmers are excellent functional molecules to attach with BNNSs, thus increasing the compatibility with nonpolar polymers (e.g., polyethylene, polypropylene, and natural rubbers). Compatible polymers and BNNSs are likely to be easily mixed, resulting in well-dispersed BNNSs within matrix. As a result, the maximum amount of load transfer could be achieved at the interphases with the lowest amount of BNNSs loading. For example, Young's modulus and tensile strength of polyacrylic acid (PAA) hydrogel (water content 70 wt.\%) composites were increased by $244 \%$ and $57 \%$, respectively, when $0.5 \mathrm{~g} \mathrm{~L}^{-1}$ BNNSs was added to the PAA hydrogel ${ }^{112}$. This excellent reinforcement 
a

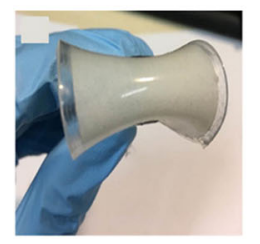

b

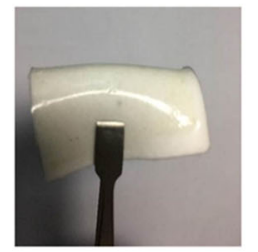

i

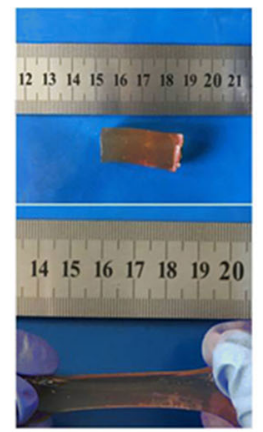

C

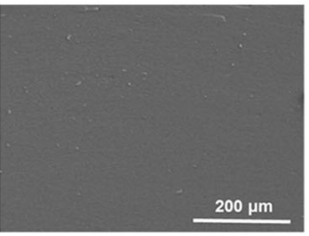

d

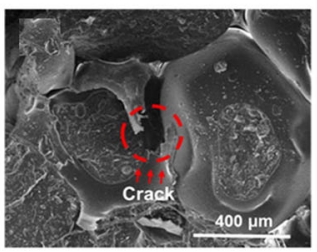

k

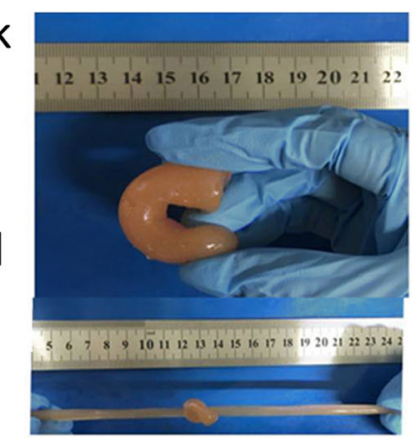

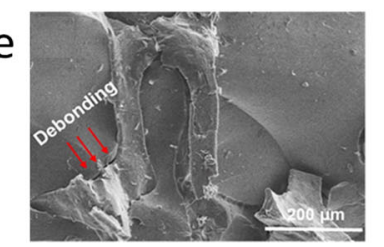

f

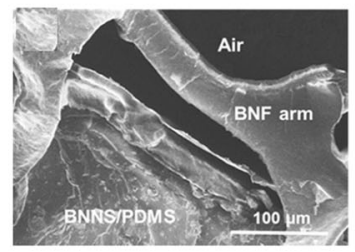

g

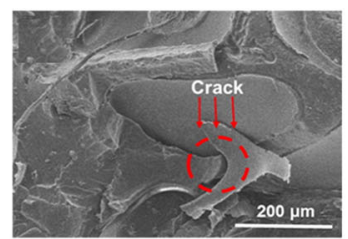

h

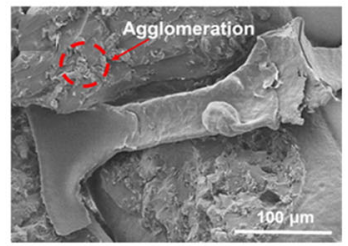

$\mathrm{m}$

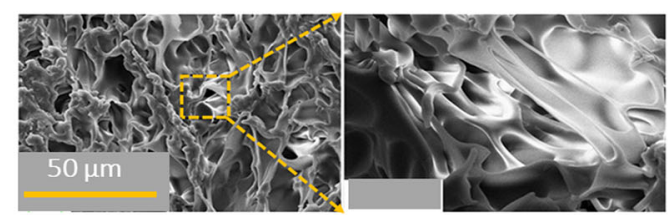

$\mathrm{n}$
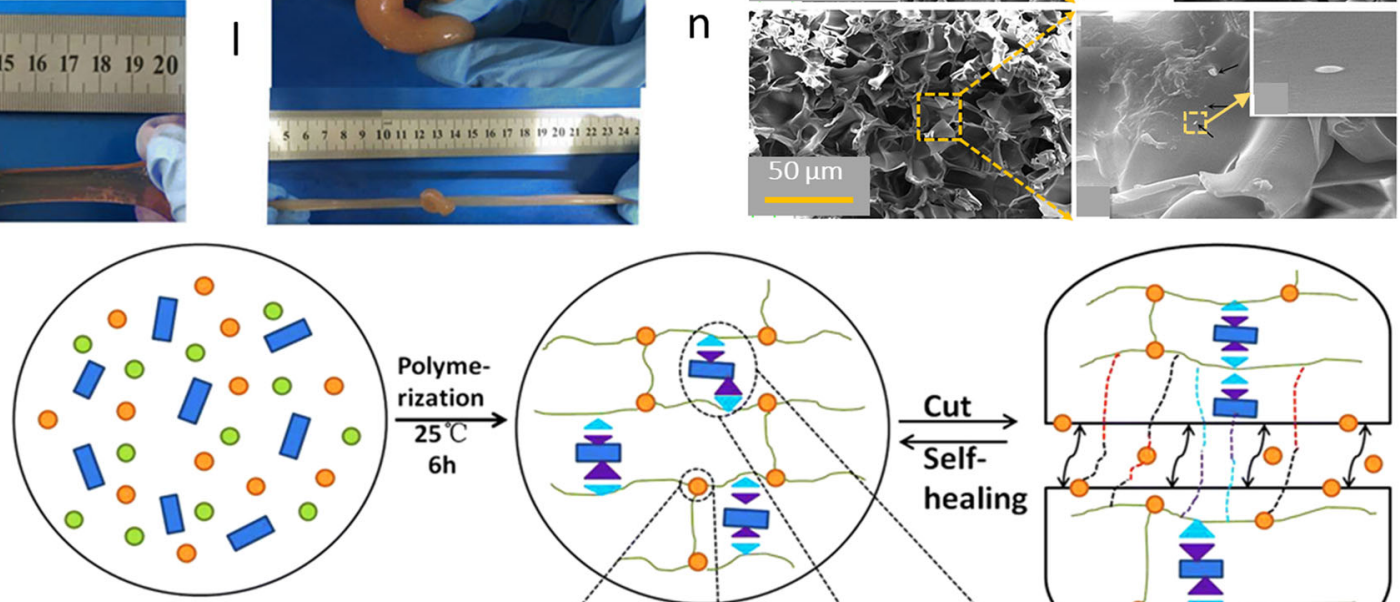

healing
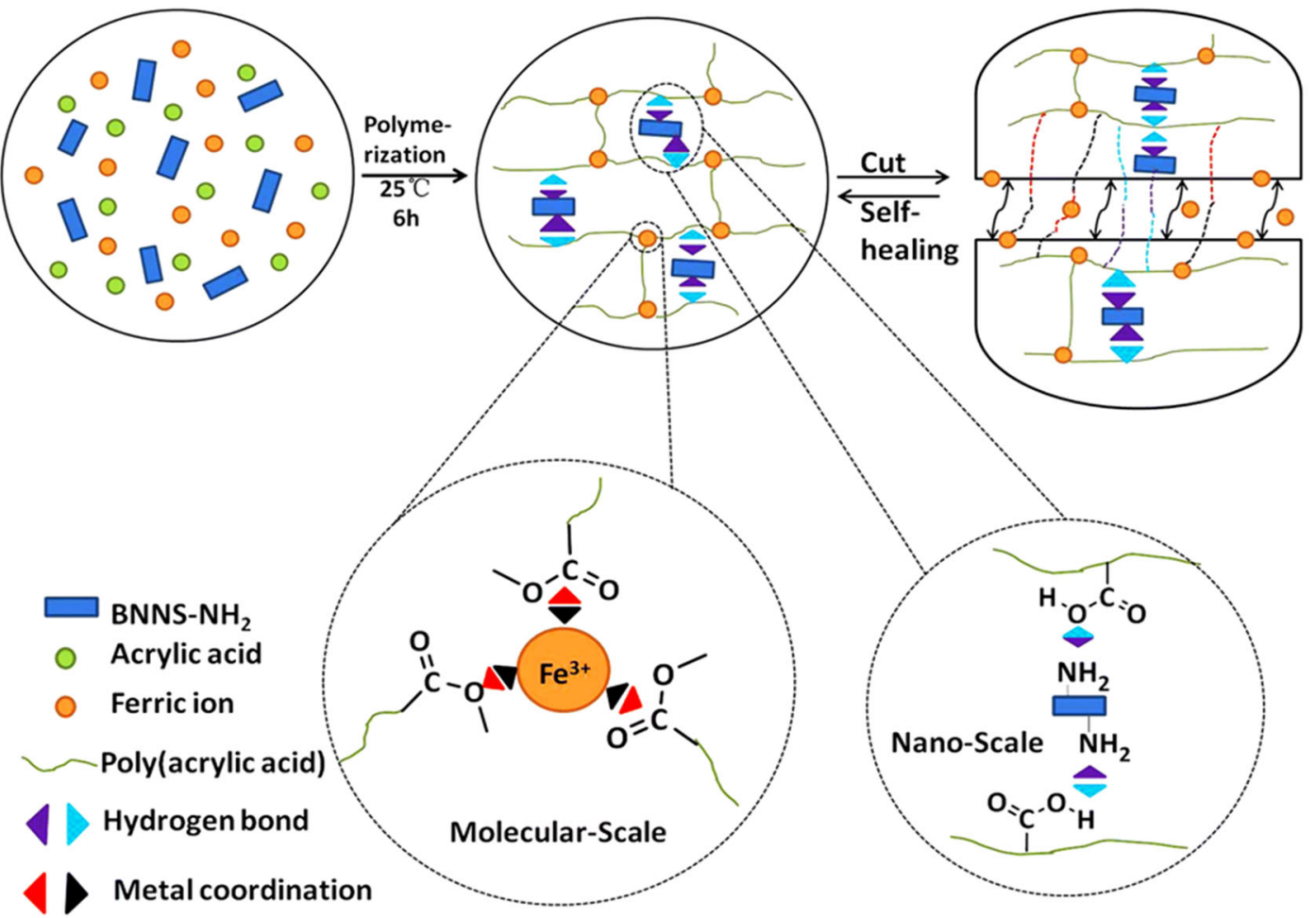

Fig. 6 Mechanical reinforcement of polymer-BNNS composite systems. Mechanical reinforcement of BNF-PDMS and BNNS-BNF-PDMS nanocomposites: $\mathbf{a}$, $\mathbf{b}$ optical photographs of flexible BNF-PDMS and BNNS-BNF-PDMS, SEM images of tensile fractured surfaces of $\mathbf{c}$ PDMS, $\mathbf{d}$ BNF-PDMS, and e-h BNNS-BNF-PDMS nanocomposites at 2, 5, 8, 10 wt.\% BNNSs loading, respectively. The digital photographs of composite hydrogels: $\mathbf{i}$ original, $\mathbf{j}$ stretched, $\mathbf{k}$ bent, and I stretched after being knotted; SEM images of $\mathbf{m}$ PAA hydrogel, $\mathbf{n} \mathrm{BNNS}^{-\mathrm{NH}_{2}}$ based PAA hydrogel composites; o schematic illustration of the formation of a PAA-BNNS- $\mathrm{NH}_{2}$ composite hydrogel with self-healing ability. a-h reprinted from ref. ${ }^{108}$, Copyright (2017), with permission from Elsevier. i-o reprinted by permission from Springer Nature: ref. ${ }^{112}$, Copyright 2018.

was achieved because of the increased compatibility of the amino group $\left(\mathrm{NH}_{2}\right)$ functionalized BNNSs with the PAA matrix. Polar-polar interaction between $\mathrm{NH}_{2}$ functional group and the PAA matrix leads to form stronger interphase by creating hydrogen bonding between them (Fig. 60). An MD simulation study reported that the polarized nature of the B-N bonds, high bonding potentials of $B$ and $N$ atoms, strong vdW and Coulomb interactions between $\mathrm{BN}$ nanosheets and polymers could be the reasons for $35 \%$ higher IFSS for BN compared with CNT composites, resulting in enhanced stress transfer at the $\mathrm{BN}$ and 
polymer matrix interface ${ }^{37}$. As a result of such an increase in IFSS, the fracture strength of the polymer-BNNS nanocomposites was improved compared with the base polymer matrix.

In addition to the factors mentioned above, several postprocessing strategies have been developed to improve the mechanical properties of the materials. For example, uniaxial and biaxial drawing of composites can result in the alignment of BNNSs within matrix. As a result, Young's modulus of ultra-high molecular weight polyethylene-BNNS composites $^{113}$ was increased by 11 times, and the tensile strength of polyvinyl chloride-BNNS composite ${ }^{102}$ was increased by $100 \%$ by such drawing operation. The chain unfolding from the surface-grown lamellae produced enough force to exfoliate the accumulated BNNSs, thus yielding a more favorable interface area for stress transfer.

Although 2D materials are effective in reinforcing the polymer materials owing to their ultra-high surface area, strong mechanical strength, and mature technology, inclusion of 2D materials into polymers yield distinct properties making them suitable for specific applications ${ }^{114}$. For example, the addition of electrically conductive graphene increases the mechanical strength and electrical conductivity of the corresponding polymer composites. On the other hand, BNNSs play a decisive role in reinforcing polymers for applications where electrical insulation is important such as thermal interface material (TIM) ${ }^{115,116}$ and load-bearing electrical insulators.

In summary, the mechanical properties (e.g., tensile modulus and strength) of polymer composites can be increased up to the theoretical limit by incorporating BNNSs. Such nanocomposites can be developed by controlling various interfacial and noninterfacial factors at different length scales, including microscopic features (voids/defects, interphases, functional groups), and macroscopic structure (aspect ratios, and mechanical interlocking). However, increasing the amount of BNNSs may lead to the agglomeration of nanosheets that act as load concentrating points and eventually reducing the load transfer efficiency. Uniformly distributed BNNSs with strong interphase and negligible defects in the polymer nanocomposites are most effective in load transfer between constituents, resulting in improved mechanical properties of the composite materials.

\section{Thermal properties}

Thermal properties of polymer-BNNS composite materials are further reviewed to assess the effect of BNNSs on the thermal conductivity, thermal stability, and $T_{\mathrm{g}}$ of polymer materials. Thermal conductivity $(k)$ is a material property that allows measurement of the ability or efficiency of heat transfer through a material. Thermal conductivity measures the vibrational energy conduction through a material by phonons or electrons. As BN does not have free electrons, phonons are the primary heat carriers in polymer-BN composite systems. In crystalline materials, thermal energy transfers from the heat source to the surface atoms, then propagates in a wave at the same speed throughout the materials. However, semi-crystalline and amorphous materials such as polymers cannot diffuse heat in the same manner because of the interface resistance ${ }^{117}$. This thermal interfacial resistance is originated from irregular polymer chain structures (amorphousness), phonon-scattering spots, and lower mean free paths. A number of theoretical studies have been conducted to demonstrate the effect of thermal interface resistance on the thermal conductivity of the polymeric composites ${ }^{118-121}$. These simulation studies demonstrate that effective thermal conductivity is improved when thermal interfacial resistances are reduced. By adding thermally conductive BNNSs into the polymer matrix, the thermal conductivity of nanocomposites can be increased exponentially (Fig. 7) ${ }^{68,109,110,122-132}$. Nevertheless, some limitations prevent polymer composites from utilizing the full potentials

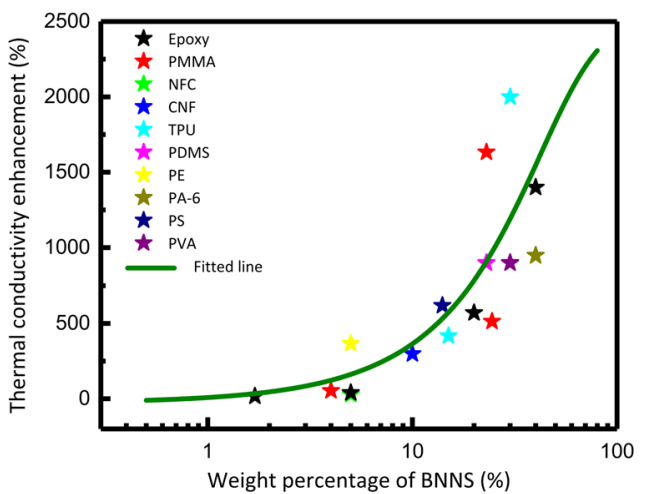

Fig. 7 Thermal conductivity of polymer-BNNS composites. An illustration of changes in thermal conductivity with varying BNNSs loading for the reported BNNS-polymer composites ${ }^{68,109,110,122-132}$. The percentage of thermal conductivity enhancement is calculated based on the neat polymer material. [PMMA polymethyl methacrylate, NFC nanofibrillated cellulose, CNF cellulose nanofiber, TPU thermoplastic polyurethane, PDMS polydimethylsiloxane, PE polyethylene, PA-6 polyamide 6, PS polystyrene, PVA polyvinyl acetate].

of BNNSs. The crucial elements that are needed to be considered for designing thermally conductive polymer composite materials can be categorized into two broad thrusts: structural factors and phonon scattering (Fig. 8).

The major obstacle in utilizing the full potentials of highly thermally conductive BNNSs into nanocomposites is phonon scattering. Figure 8 demonstrates several phonon scattering that is usually occurred in polymer composites, including (i) phononphonon scattering, (ii) phonon-interface scattering, and (iii) phonon-defect scattering ${ }^{43}$. The phonon scattering can be reduced in several ways, such as creating a strong/immobile interphase, increasing filler geometry, creating a 3D network, and aligning BNNSs within the nanocomposite matrix ${ }^{43,76}$.

Functionalization is one of the several techniques that have been utilized to minimize these scattering by strengthening interphases. For instance, Huo et al. ${ }^{133}$ increased the thermal conductivity of epoxy-BNNS composites by $513.5 \%$ when modifying BNNSs with coupling agent silane. This increase was attributed to the reduction in thermal resistance by strengthening the interphase between BNNSs and epoxy. Thermal resistance could be further reduced by increasing the bonding strength or molecular interaction type. Such results were demonstrated for the epoxy-BNNP composites utilizing stronger chemical bonding, i.e., covalent (BNNP-HBP) and weaker chemical bonding, i.e., noncovalent functionalized BNNP (BNNP-ODA) ${ }^{134}$. BNNP-HBP composites exhibited a thermal conductivity increase of 40 -fold, whereas BNNP-ODA showed an increase of 35 -fold. The BNNP-HBP creates a stronger molecular interaction with the epoxy matrix compared with BNNP-ODA, resulting in better thermal conductivity.

Increasing interfacial surface area and filler geometry also play a crucial role in shortening thermal conductivity paths. As such, the thermal conductivity of PMMA-BNNS was increased by $\sim 1700 \%$ for $140 \mathrm{~m}^{2} \mathrm{~g}^{-1}$ surface area of BNNSs ${ }^{68}$ as opposed to $\sim 60 \%$ for $6.77 \mathrm{~m}^{2} \mathrm{~g}^{-1}$ surface area of BNNSs ${ }^{127}$ at the same amount of loading ${ }^{127}$. As heat fluxes are transferred from polymer matrices to BNNSs through the interfaces, increasing interfacial surface area facilitates the transfer of a higher amount of heat from matrix to nanosheets. As a result, exfoliation and well-dispersion of BNNSs are very effective in increasing the thermal conductivity ${ }^{131,135}$.

Phonon scattering is highly dependent on the irregularity, interface structures, and defects of the nanocomposites. Therefore, structural factors such as polymer chain organization, shape, size, and orientation of BNNSs regulate the heat conduction paths of the materials ${ }^{50}$. For instance, polymers of higher crystallinity, such as high-density polyethylene, are highly thermally 


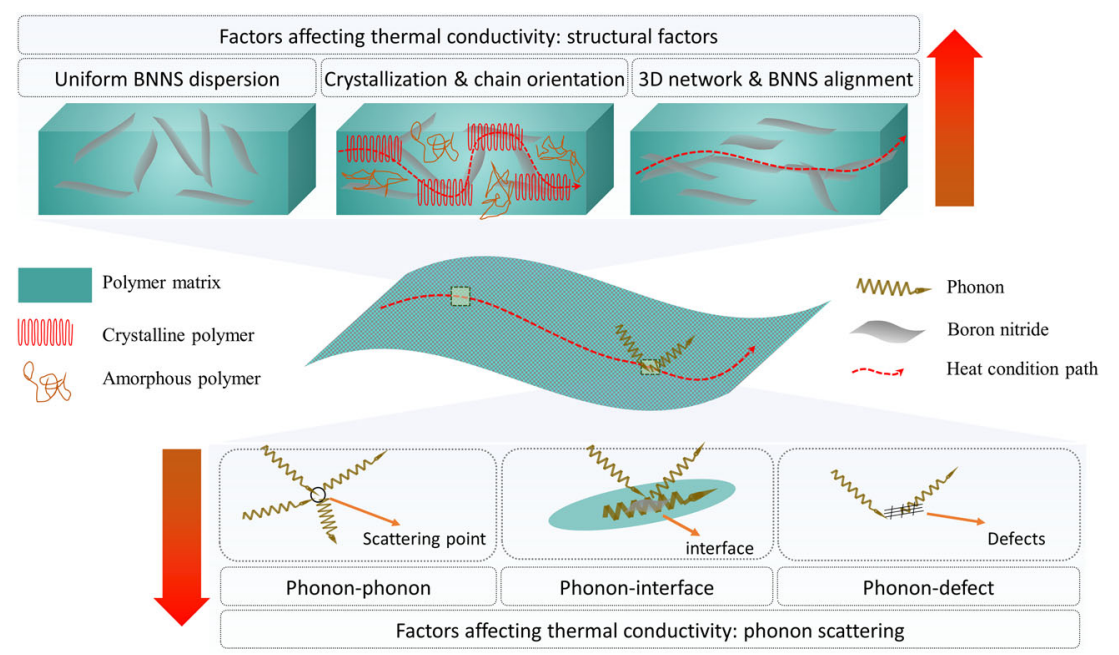

Fig. 8 Illustration of the factors affecting the thermal conductivity of BNNSs incorporated polymer nanocomposite systems. Structural factors of polymer nanocomposites include BNNSs dispersion, crystallization \& chain orientation, and 3D network and BNNSs alignment ${ }^{50}$. Phonon scattering in polymer nanocomposites includes phonon-phonon scattering (such as random polymer chain scattering), phononinterface scattering (such as polymer-BN interface), and phonon-defect scattering (such as voids) ${ }^{43}$. Red arrows indicate desired change (i.e., increase or decrease) of the corresponding factors to improve thermal conductivity of the polymer nanocomposites.

conductive $\left(0.5 \mathrm{~W} \mathrm{~m}^{-1} \mathrm{~K}^{-1}\right)$, whereas amorphous-rich polymers, like low-density polyethylene, show low thermal conductivity $\left(0.26 \mathrm{~W} \mathrm{~m}^{-1} \mathrm{~K}^{-1}\right)^{136}$. Interestingly, BNNSs can act as a nucleating point and increase crystallinity. Li et al. ${ }^{110}$ reported that incorporating BNNSs increased crystallinity of the polymers from 36 to $38 \%$, therefore increasing the thermal conductivity by $154 \%$. High aspect ratio and lateral dimension of thermally conductive 2D nanosheets are also likely to reduce the interface numbers, therefore reducing phonon scattering and increasing mean free paths for heat conduction ${ }^{137}$.

A well-structured and interconnected 3D network of BNNSs can further reduce phonon scattering, originated from structural defects of amorphous polymers (Fig. 8$)^{43}$. Several techniques have been explored to create a 3D network of BNNSs, including tape casting ${ }^{138}$, spin-casting ${ }^{139}$, applying shear force $e^{140}$, mechanical stretching ${ }^{140}$, employing magnetic force ${ }^{141}$, or electric fields ${ }^{142}$. For example, Lin et al. ${ }^{101}$ modified the BNNSs surface by imparting iron oxide and aligned the BNNSs by applying a magnetic field. Thermal conductivity was increased 5.7 -fold by adding vertically aligned BNNSs while randomly oriented BNNSs improved that by 2.8 -fold at the same $20 \mathrm{wt}$. $\%$ loading amount. Because of the anisotropic nature of thermal conductivity, heat can transfer faster along the in-plane direction than the throughplane direction of BNNSs. As a result, aligning the BNNSs within nanocomposites and creating an interconnected BNNSs network could increase the heat conductivity by higher values than randomly oriented BNNS composites.

It is interesting to note that the experimental measurement of thermal conductivity of polymer-BNNS composites could be different than the predicted thermal conductivity evaluated by simulation studies. For example, Lin and co-workers showed that the predicted enhancement of thermal conductivity of epoxyBNNS composites is $\sim 32 \%$ while experimental values showed an enhancement of $104 \%^{127}$. This discrepancy was explained considering several factors: (i) the complexity of real composite systems when compared with theoretical calculation such as BNNSs size, shape, and thickness. Such complexity of the system could lead the calculation to underestimate or overestimate thermal conductivities of the polymer composite materials, (ii) thermal boundary resistance (TBR) values could not be measured experimentally, therefore, TBR values are assumed in theoretical calculation, resulting in an error in predicted values, (iii) because of the anisotropic nature of BNNSs, the assumed thermal conductivity of BNNSs are either lower or higher in theoretical calculation than the actual thermal conductivity of BNNSs, and (iv) experimental error, originated from either method or procedures, could also contribute to the discrepancies between theoretical and experimental results ${ }^{127,143,144}$.

He et al. ${ }^{145}$ developed a ternary composite via a hightemperature pressing method to reduce void formation. The high-temperature pressing method reduces the structural defects/ voids originating from low dense packing and microstructural defects (such as grain boundaries, stacking faults). These defects are occupied by air, resulting in reduced thermal conductivity. As a result of reducing defects, lower phonon scattering and higher thermal conductivity are achieved compared with the constituent binary composites.

Thermal stability of a material is the ability to resist or delay the degradation of molecular structures owing to heating. For polymer materials, the chemical structure consists of long-chains and short-chains of polymer molecules that are bonded with weak vdW forces. As a result, these polymer materials are susceptible to easy degradation under heating. Polymer usually decomposes into different volatile gases and produces chars either in the presence of oxygen (oxidative degradation) or in the absence of oxygen (pyrolytic degradation) ${ }^{146}$. Nanofillers may reduce these thermal degradation processes by acting as a mass barrier and catalyst $^{147,148}$. 2D materials such as BNNSs can reduce the diffusion and transmission rate of oxygen and decomposed volatile gases within the nanocomposite matrix, thus slowing down the decomposition reaction rate (Fig. 9) ${ }^{149-151}$.

Because of the morphology, 2D nanosheets are more effective as a gas barrier than any other shapes such as $0 D, 1 D$, and bulk materials ${ }^{152-154}$. Owing to the impermeable nature of $2 \mathrm{D}$ nanosheets, both volatile and oxygen gases have to diffuse around BNNSs instead of taking straight paths within polymer nanocomposites (Fig. 9). Thus, the mean free paths of gas diffusion through the polymer nanocomposites are increased, leading to a delay in the material degradation process. The barrier property of BNNSs was illustrated for polyacrylonitrile (PAN) composite systems (PAN-10 wt.\% BNNS), where a 15\% improvement in thermal stability (5 wt.\% mass loss temperature) was reported $^{155}$. Increasing the surface area of BNNSs would impede the gas flow even higher, resulting in more enhancement in thermal stability. Mesoporous BNNSs have a higher specific surface area $\left(140 \mathrm{~m}^{2} \mathrm{~g}^{-1}\right)$ compared with conventional BNNSs 


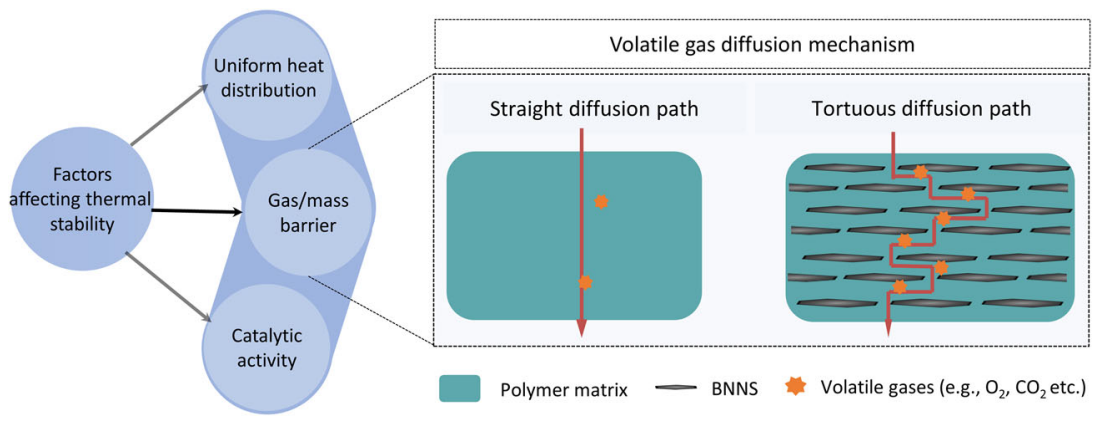

Fig. 9 Factors affecting thermal stability of polymer-BNNS composites. Schematic illustration of factors affecting the thermal degradation of polymer-BNNS composites. Straight diffusion/migration paths used by volatile gases and oxygen molecules for polymer matrix result in fast thermal degradation. In contrast, BNNSs create tortuous paths within the matrix; therefore, volatiles and oxygen gases are required to pass around BNNSs, resulting in delayed thermal degradation ${ }^{149-151}$.

$\left(27 \sim 50 \mathrm{~m}^{2} \mathrm{~g}^{-1}\right)$, resulting in $\sim 35 \%$ improvement in thermal stability (10 wt.\% mass loss temperature) compared with pure PMMA $^{68}$. These mesoporous structures can trap the mobile gases and reactive molecules; therefore, the transmission rate of volatile gases, free radicals, and oxygen molecules can significantly be reduced. Although volatile gas reduction could delay the weight loss of the materials, free radicals and oxygen reduction would obstruct the material degradation processes.

Uniform distribution of BNNSs into polymers is vital to disseminate the heat; otherwise, local hotspots can be created from agglomerated BNNSs. For instance, the thermal stability of thermoplastic polyurethane (TPU)-BNNS composites was reduced from 347 to $343{ }^{\circ} \mathrm{C}$ for $10 \%$ weight loss temperature because of BNNS agglomeration. Agglomerated BNNSs act as a heat concentrating point, thus expediting the thermal degradation process compared to neat polymers. Such results were also demonstrated for other polymers, including thiol-epoxy ${ }^{156}$, epoxy $^{133}$, polyimide $(\mathrm{PI})^{157}$, polystyrene $(\mathrm{PS})^{158}$, polybenzimidazole $^{105}$, and photosensitive polyimide ${ }^{159}$ composite systems.

Flame retardancy, usually evaluated by measuring the limiting oxygen index (LOI), is another crucial parameter that gives insights into the thermal stability of a material. LOI indicates the minimum amount of oxygen concentration needed for polymer combustion. Adding BNNSs into polymer materials increases LOI, resulting in a higher amount of oxygen required for polymer combustion. A PAN-based polymer nanocomposite (PAN-BNNS) reported an increment of LOI from 18 to $27 \%$ because of $10 \mathrm{wt} . \%$ BNNSs addition $^{155}$.

On top of the barrier properties, nanofillers, modifiers, or any other decomposed products (chars) may exhibit catalytic effects. As a result, thermal decomposition reaction kinetics could be changed/modified by altering the activation energy and reaction mechanisms ${ }^{160-167}$. It is possible to impart catalytic functionalities by attaching BNNSs with appropriate functional molecules. Thus, thermal degradation mechanisms such as rate-limiting steps, reaction kinetics could be changed accordingly. To the best of our knowledge, the catalytic behavior of BNNSs for thermal decomposition of polymer nanocomposites has not been reported yet.

$T_{\mathrm{g}}$ of amorphous or semi-crystalline polymer materials indicates a gradual transformation from a brittle or glassy state into a viscous or rubbery state. The $T_{\mathrm{g}}$ can give fundamental insights into the polymer chain dynamics and helps to explore more areas of application. For example, materials with below room temperature $T_{\mathrm{g}}$ indicate pliable application, whereas materials with higher than room temperature $T_{\mathrm{g}}$ indicate a rigid application. In the molecular scale, various nanoscale chain dynamics such as atom translational mobility, bond or segmental reorientation dynamics, torsional dynamics, and dynamic heterogeneity play a crucial role in influencing $T_{\mathrm{g}}{ }^{168}$. As a result, polymer chain interaction regulates the chain dynamics of materials by either attractive or repulsive force ${ }^{169-178}$. Although attractive force leads to an increase in $T_{\mathrm{g}}$ repulsive force gives rise in chain mobility and accelerates structural relaxation ${ }^{179-182}$.

$2 \mathrm{D}$ materials such as BNNSs have effects on polymer chain mobility because of the mechanical interlocking characteristics and molecular interactions at the interphase regions ${ }^{161}$. The mechanical interlocking characteristic increases activation energy for polymer chain movement and reduces chain mobility within nanocomposite matrix ${ }^{183}$. Increasing the amount of BNNSs would effectively raise the activation energy of the chain mobility, thus hiking $T_{\mathrm{g}}$. Such result was demonstrated for the PMMA composite matrix where $T_{\mathrm{g}}$ was increased from 3 to $13^{\circ} \mathrm{C}$ when the amount of BNNSs was increased from 0.3 to 15 wt. $\%{ }^{68,183}$.

Aside from physical interaction originated from BNNSs interlocking with polymer chains, molecular interactions can also be forged between constituent nanocomposites to engineer the $T_{\mathrm{g}}$. By controlling the molecular interactions, immobile interphase at the BNNS-polymer interface can be developed ${ }^{133}$. For example, BNNP was functionalized in both covalent (BNNP-HBP) and noncovalent (BNNP-ODA) methods and utilized to fabricate epoxyBNNP $^{49}$. As covalent bonding is more robust than non-covalent bonding, a reduction in chain mobility by BNNP-HBP was observed to be higher compared with BNNP-ODA. As a result, the $T_{\mathrm{g}}$ of BNNP-BHP composites was increased to higher values. Such effect of functionalization was also investigated by MD simulations, where the predicted change in $T_{\mathrm{g}}$ of PMMA composites agreed well with the experimental results ${ }^{184}$. These simulation studies indicate that the polymer-nanofillers interactions have an important role on the polymer chain mobility, resulting in increased or decreased $T_{\mathrm{g}}{ }^{182}$.

As $T_{\mathrm{g}}$ depends on local chain mobility, the higher heat transport should prevent local hotspots formation; otherwise, local polymer chains at the hotspots would move quickly ${ }^{185}$. Local hotspots (or heat concentrating points) are common consequence of BNNSs agglomeration. Detailed discussion to avoid heat concentrating points is explained in the thermal conductivity section of this article. Research reported degradation in $T_{\mathrm{g}}$ for TPU-BNNS composites by $23 \%$ (compared with TPU) ${ }^{186}$ and PS-BNNS composites by $\sim 4 \%$ (compared with PS) ${ }^{158}$ because of BNNSs agglomeration.

2D materials with high thermal conductivity are poised to potentially solve the increasingly high heat dissipation problems that are common in electronic devices. For example, graphene and $\mathrm{WS}_{2}$ incorporated polymer nanocomposites with very high thermal conductivity can be utilized to distribute the heat of energy storage devices ${ }^{187-189}$. Unfortunately, such nanocomposites also showed increased electronic conductivity that is detrimental for applications like TIM and battery electrolytes or battery packaging. In such cases, BNNSs incorporated polymer nanocomposites are ideal ${ }^{190}$. 
In summary, the thermal properties of polymer materials can be improved through appropriate assemblies of BNNSs into polymer materials as such by controlling BNNSs loading, dispersion, orientation, and void/defect formation within nanocomposites. The structure, regularity, and interface of the polymer matrix and thermally conductive BNNSs play a key role in the thermal conductivity value of the final polymer composites. However, phonon scattering is a limiting factor toward developing thermally conductive composite materials. Fortunately, well dispersion of BNNSs and strengthened interphase between BNNSs and polymer matrix potentially minimize the phonon scattering. Interconnected networks of BNNSs with strong interphase and minimized agglomerations are most effective in developing thermally conductive nanocomposites. On the other hand, the barrier and catalytic properties of BNNSs could further reduce the volatile gas flow of the polymer nanocomposites during thermal degradation process and increase the thermal stability of the composite materials.

\section{Electrical and electrochemical properties}

Applications such as power electronics use high dielectric polymer materials that show promising reliability and higher voltage breakdown strength ${ }^{191,192}$. However, these materials fail to perform well at extreme conditions such as at high temperatures $^{193}$. Polymer nanocomposites could be configured to address such problems by incorporating the merits of BNNSs and polymers. For example, Wang et al. ${ }^{194}$ developed BNNSs incorporated divinyltetramethyldisiloxane-bis(benzocyclobutene) (CBCB) nanocomposites for dielectric application at high temperatures. At high-temperature $\left(70^{\circ} \mathrm{C}\right)$ operation, dielectric losses of conventional polymers were increased from 15 to $76 \%$. On the contrary, BNNSs incorporated $C B C B$ composites showed surprisingly very low increment (dielectric loss was increased from 15 to $18 \%$ at $70^{\circ} \mathrm{C}$.

High electrical resistivity and dielectric breakdown strength are critical for many applications, including TIM and microwave electronic packaging. Because of the high bandgap in $\mathrm{BN}$ materials, the addition of BN nanomaterials into the polymer matrix does not increase the electrical resistivity of polymers. There have been reports on no change in electrical conductivity for BNNSs incorporated PMMA, polyvinyl butyral, PS, poly (ethylene-vinyl acetate) ${ }^{109,126}$. In addition, the voltage breakdown strength of epoxy matrix increased from $\sim 251 \mathrm{kV} \mathrm{mm}^{-1}$ (for pristine epoxy) to $\sim 260,281,242$, and $176 \mathrm{kV} \mathrm{mm}^{-1}$ in the nanocomposites containing $0.2,0.6,1$, and 5 vol.\% of $\mathrm{BN}$ nanomaterials, respectively ${ }^{195}$. The improvement of breakdown strength at low loading could be attributed to the lower dielectric loss and higher thermal conductivity. In contrast, the decreased breakdown strength at higher loading levels could be attributed to the possibility of the presence of structural imperfections. Such structural imperfections are resulted from the agglomeration of BNNSs, thus increasing the possibility of void formation around nanosheets ${ }^{195}$. It is important to create strong interphase and uniform dispersion of BNNSs into polymer matrix in order to improve the breakdown strength ${ }^{196}$. For example, Xing et al. ${ }^{197}$ fabricated polymer nanocomposites by incorporating functionalized BNNSs. The authors reported a monotonic increase in both dielectric breakdown strength and electrical resistivity with increasing BNNSs loading up to 8 vol.\%. Such results indicate the importance of creating strong interphase and uniform dispersion of BNNSs in order to develop electrically insulative and high dielectric strength materials.

Lithium battery technology is yet another emerging domain that is growing fast to address pollutant emission, independence to fossil fuel resources, and global warming concerns. However, this requires tackling critical challenges such as poor thermal conductivity of battery materials, low ionic conductivity of electrolytes, and lack of sufficient mechanical robustness in separators to suppress lithium (Li) dendrites, leading to battery failure and exothermic thermal runaways. Chen et al. ${ }^{198}$ demonstrated that the thermal conductivity of electrolytes with BNNSs could be increased up to 50 times compared with pristine electrolytes. Also, such composites exhibited reduced dielectric loss, increased electrical resistivity, and enhanced voltage breakdown strength that enable them to be promising for cooling applications. Shim et al. ${ }^{199}$ substantially improved ionic conductivity, $\mathrm{Li}+$ ion transference number, and mechanical properties (i.e., modulus) of gel-electrolytes using a very low amount of functionalized boron nitride nanoflake (BNNF) (0.5 wt.\%). Because of the Lewis acid-base interaction, BNNFs increased $\mathrm{Li}+$ ion conduction by trapping the anions of the electrolytes. Besides, $\mathrm{Li}+$ ion can also be transported through the segmental motion of the electrolytic polymer matrices, and such segmental motion depends on the amorphous portion of the polymer materials ${ }^{200}$. In such cases, nanofillers such as BNNSs could be utilized to increase the amorphous portion of the polymers (i.e., plasticizing effect), therefore increasing the ionic conductivity of polymer electrolyte materials $^{201}$. Waqas et al. ${ }^{202}$ fabricated a bi-layer PE-BN/poly (vinylidene flouride-co-hexagluoropropene) (PVDF-HFP) separator that significantly suppressed lithium dendrite growth owing to the great adsorption energy with polymers, large interactive surface area, and superior mechanical properties (i.e., modulus).

2D materials such as graphene, MXene, and $\mathrm{MoS}_{2}$, are shown to be good candidates for Li batteries as cathode, anode, and catalyst materials ${ }^{203}$. However, these $2 \mathrm{D}$ materials are not suitable for the battery electrolyte or packaging applications since increased electronic conductivity would fail the battery by short circuiting. In such cases, BNNSs have advantages over other 2D materials (graphene, MXene, and $\mathrm{MoS}_{2}$ ) where electronic insulation properties are crucial ${ }^{190}$. In summary, the plasticizing effect and Lewis acid-base characteristic of BNNS may facilitate to $\mathrm{Li}^{+}$ion conduction through the polymer electrolytes, resulting in increased ionic conductivity of Li batteries. In addition, thermally conductive BNNSs are shown to distribute the heat generated within the electrolytes during battery operation, resulting in the reduced hot-spot formation and avoiding thermal run-away events $^{190}$.

\section{Optical properties}

The interest for the optical properties of polymer materials is increasing in order to design transparent polymer nanocomposites that could exhibit properties such as light absorption (UV, infrared, and visible light), UV photodetion, photoluminescence, dichroism, or non-linear optical properties ${ }^{204}$. BNNSs possess important optical properties such as UV-vis absorption, deep UV and thermal neutron detection, and photoluminescence ${ }^{205-207}$. The optical properties of BNNSs make them a suitable choice as nanofillers for the fabrication of polymer composites that are likely to develop visible light transparent, UV-blocking with photoluminescence properties into polymer materials ${ }^{208}$.

The main factors affecting the optical properties of nanocomposites include the electronic nature, size, and spatial distribution of the BN nanosheets within polymer matrices. Although the intrinsic optical properties of BNNSs such as color absorption and emission depend on the nanosheets size, the spatial distribution of the nanosheets has a major role in configuring the optical properties of the materials ${ }^{204}$. Uniform distribution of nanosheets promotes optical transparency, photoluminescence, UV, and IR absorption while an ordered distribution may favor iridescence, and a uniaxially oriented distribution of nanosheets may favor dichroism ${ }^{204}$. For example, Min et al. ${ }^{81}$ incorporated BN nanoparticles and nanosheets into PI and investigated the optical properties of corresponding nanocomposites. The PI-BN nanosheets composites possessed high transparency at the 
wavelength of $550 \mathrm{~nm}$ (75.4\% transmittance), as opposed to PI-BN nanoparticles composites ( $0.1 \%$ transmittance) at the same $10 \mathrm{wt}$. $\%$ BN loading. This indicates that the opaque nature of the BN particles decreased the transparency of the composites (UV-vis properties of the polymer nanocomposites). Interestingly, the transparency properties of BNNS composites were not changed much (transmittance of base PI and PI with 3 wt.\% BNNS are $88 \%$ and $84 \%$, respectively) up to the addition of 3 wt.\% BNNS, indicating the uniform distribution of nanosheets within nanocomposite matrix. The addition of more BNNSs (i.e., $10 \mathrm{wt} \%$ ) decreased the transparency properties of PI-BNNS composites to $75.4 \%$. As the concentrations of BNNSs increase, the tendency to agglomerate also increases. Such agglomerates act as larger particles, therefore blocking and scattering the visible light that are passing through. In addition, BNNSs also showed intense cathodoluminescence emission properties, indicating the potential use of BNNS nanocomposites as UV laser emitters ${ }^{209}$. The other optical properties of BNNS such as deep UV photodetectors are interesting because of their military applications such as missile launching detection, gunshot warning, UV flame sensing, and secure space communications ${ }^{210}$.

The need for UV-protective materials is in great demand owing to the thinning of the atmospheric ozone layer. The BNNS is considered as one of the promising UV-filters that can be incorporated into polymer materials to modify the UV-filtering properties of the composites ${ }^{204}$. For example, Bisht et al. ${ }^{211}$ investigated the PMMA composites using amine-functionalized BN nanoflakes where $0.5 \mathrm{wt} . \%$ BN nanoflakes incorporated PMMA composites shielded UV-wavelength (UV-B and UV-C) by $325 \%$ while retaining high transparency of $65 \%$ to visible light. However, increasing the BN nanoflakes loading improves the UV-blocking properties as well as reduces the transparency of the nanocomposites.

Although 2D materials such as graphene are effective in improving the optical properties as such absorbing visible lights, UV-near infrared light, and shielding UV-radiation, such nanocomposites are likely to have high electronic conductivity that is not desirable in certain opto-electric applications ${ }^{212,213}$. In such cases, the incorporation of BNNSs is likely to be advantageous as the bandgap of $\mathrm{BN}$ is much higher $(5.7 \mathrm{eV})$ compared with graphene.

\section{BNNS-POLYMER NANOCOMPOSITE APPLICATIONS}

Polymeric composite materials are in demand for many emerging applications in consumer electronics, energy storage, and mobility systems. Although there are many nanofillers that can be added to polymer materials, the authors believe that BN nanosheets polymer composites are suited for applications where a combination of very low electrical conductivity, high thermal conductivity, high mechanical properties, and high electrical breakdown is needed. Conventional 2D nanofillers such as graphene and MXene are electrically conductive ${ }^{26-29}$, therefore the addition of such nanofillers could increase the electrical conductivity of the nanocomposites. BNNS-polymer nanocomposite materials are suitable for applications where high electrical resistivity is critical as seen in TIM, electrical cable insulation, and battery electrolytes. In addition to high electrical resistivity, such applications require high heat dissipation to avoid local hotspots generation from daily operation. The high thermal conductivity of BNNS is known to increase the heat dissipation capability and reduce the hotspots generation in polymer materials that inherently have very low thermal conductivity. Figure 10 indicates some of the promising applications of BNNS-based materials. Electronic devices often need materials (such as TIM) that can distribute heat efficiently since local hotspots can be created under service operations that may lead to performance and reliability degradation of the devices. Thermally conductive materials and, in particular, BNNSs

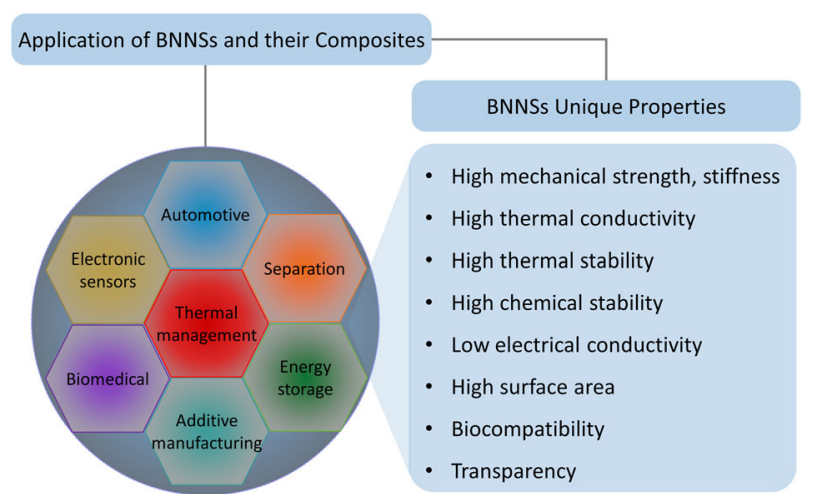

Fig. 10 Application of BNNS-based materials. Illustration of unique properties of BNNSs and their application in the emerging fields. High thermal conductivity and electrical resistivity are required for thermal management and electronic sensors ${ }^{214,215}$. Mechanical strength and stiffness are good for structural material application ${ }^{218}$. Biocompatibility is important for manufacturing biomaterials ${ }^{224}$. Electronic resistivity but ionic conductivity are unique properties of BNNS and important for energy storage application ${ }^{199,222,226,227}$. Besides, transparent properties of few layer BNNSs are crucial for optical application ${ }^{208}$.

are promising for such applications because of high electrical resistivity and thermal conductivity ${ }^{214,215}$. For example, electronic packaging is one important area of application where higher thermal conductivity is required while maintaining electrical insulation ${ }^{101,214}$. In such cases, BNNSs have the combination of both thermally conductive and electrically insulative properties to improve the polymer materials performance. Sophisticated electronics such as hazardous chemical detectors (these detectors use abundant $\Pi$ electron clouds of BNNS to detect different chemical species) ${ }^{216}$, temperature sensors ${ }^{217}$, and coatings in electronics ${ }^{214}$ are some other areas of such applications.

The mobility industry, such as automotive and aircraft, require lightweight structural materials with high strength, stiffness, and thermal conductivity. As for example, parts such as fuel tank and engine cover require very high heat management, cable insulation and sheathing require electrical insulation, wheels and tires require good tribological properties ${ }^{218}$.

Technological improvement in renewable energy demands high-performance energy storage devices that can perform under extreme weather conditions ${ }^{219,220}$. Recent work demonstrated a BNNS composite membrane separator for batteries that can withstand up to $150{ }^{\circ} \mathrm{C}^{221}$. Besides, the Lewis acidic characteristic of BNNS is shown to increase the $\mathrm{Li}^{+}$ion mobility, and higher thermal conductivity of BNNS is shown to lead to homogenous thermal distribution and the suppression of dendritic $\mathrm{Li}$ growth $^{199,222}$. Thus, thermally safe, and long cycle batteries can be manufactured in the future for energy storage devices used in electric vehicles and grid-scale energy storage systems ${ }^{223}$. Because of such properties of BNNS, many applications, including biomaterials $^{224}$, sorbents ${ }^{217,225}$, and membranes for water and gas separation ${ }^{226}$, fire-resistant coatings ${ }^{217}$, and catalytic support materials have been utilizing BNNSs for their material development ${ }^{227}$.

To summarize the status of commercial development for BNNSs-polymer composites, an estimated timeline is illustrated in Fig. 11. The key properties of BNNSs including mechanical strength, flexibility, electrical resistivity, optical, and thermal conductivity play a decisive role in the application and commercialization of such products. The most promising applications include electronic and energy materials where insulation and fast heat distribution are required such as TIM, electronic 


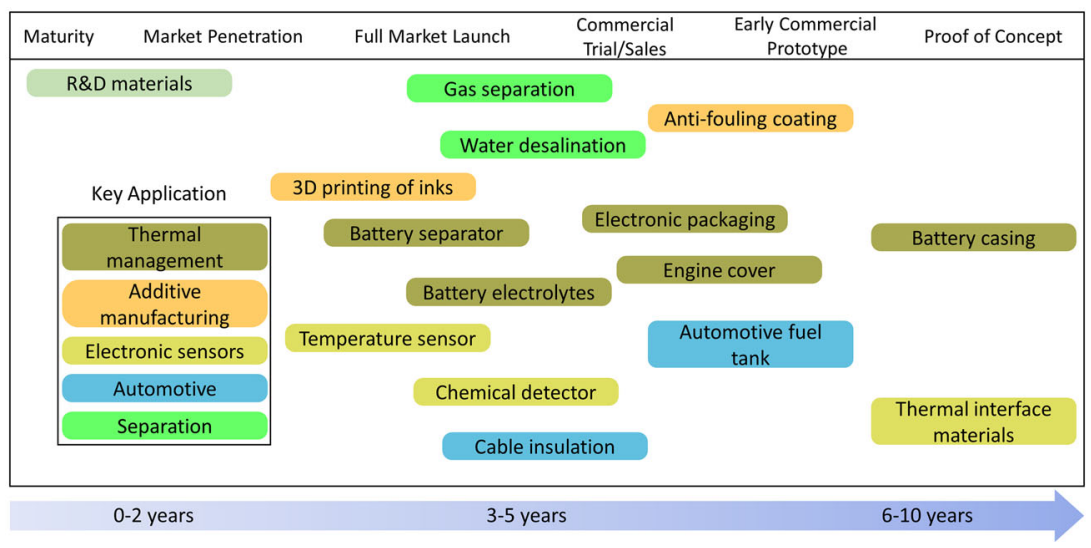

Fig. 11 Development stages of BNNS-based materials commercialization. Different stages of development of BNNS materials and their composites, and estimated timeline for their commercialization $24,25,39-43,50,90,111,253,254$.

packaging, electronic sensors, cable insulations, battery electrolytes, and battery packaging.

\section{CONCLUSION AND FUTURE OUTLOOK}

In the past decades, nanocomposite engineering has been explored to address the low mechanical properties, thermomechanical stability, and thermal conductivity of polymer materials. Particularly, BNNS nanofillers show good potential in electronic and insulation application owing to their exceptional mechanical properties, thermal conductivity, structural stability, and electrical resistivity. However, there are many remaining challenges to develop high-performance BNNS-polymer nanocomposites as such low chemical reactivity of BNNSs, weak molecular interactions between BNNSs and their host polymers, and nonuniform dispersion of BNNSs. Therefore, innovations in BNNS-polymer nanocomposites are still needed to translate the excellent material properties of BNNSs into polymer materials.

This work highlights the design principles of BNNS-polymer composites by reviewing both interfacial (i.e., covalent, and noncovalent interaction, interphase, and interfacial surface area) and non-interfacial (i.e., mechanical interlocking, dispersibility, defects/ interface space, and processing parameters) factors. These factors are shown to be major players for controlling the mechanical, thermal, electrical, and optical properties of polymers. The BNNSpolymer composites properties can be increased to the theoretical limits by controlling these factors at different length scales, including microscopic and macroscopic levels. Mechanical properties are affected by the effective load transfer between nanosheets and polymer matrix requiring uniform distribution, strong interphase, and negligible defects. However, agglomeration of BNNSs is an obstacle in achieving uniform dispersion, and such agglomerates may act as stress concentrating points, resulting in low mechanical properties and optical transparency. On the other hand, phonon scattering is the limiting factor toward developing thermally conductive composite materials. The phonon scattering could be minimized by reducing defects, strengthening interphase, and creating a network of BNNSs that can act as a thermally conductive path for conducting heat. In addition, the structure and regularity of polymer chains and BNNSs, and their interfaces play a major role in controlling the thermal conductivity of polymer composites. The interphase between BNNSs and polymer matrix has a crucial role in transferring the mechanical stress, thermal heat, and electrical properties from one phase to another. The interphase strength can be increased by forming physical and chemical bonds such as $\mathrm{H}$-bond, vdW force, and $\pi-\pi$ interactions between BNNSs and polymers, thus facilitating an effective load transfer at the interphases. This could be achieved by activating the chemically-inert BNNSs via functionalization.

The functionalization of BNNSs using covalent and non-covalent bonds and their effects on the structure-property relationship of composites are discussed. Chemical compatibility between polymers and BNNSs is vital for achieving mechanically strong and thermo-mechanically stable composites. For example, BNNSs attached with oxide and hydroxide functional groups are compatible with polymers such as polyvinyl acetate (PVA), PAN, PMMA, PAA owing to the polar-polar interactions. In contrast, silane coupling agents develop both polar and nonpolar functionalities and are compatible with either polar or nonpolar types of polymer materials. Such strategies can enhance the polymer adhesion with BNNSs and reduce polymer chain dynamics. A reduction in chain dynamics increases storage modulus, $T_{\mathrm{g}}$, and dimensional stability of the composites. Furthermore, a homogenous dispersion of monolayer BNNSs is critical for increasing the molecular interactions of polymer chains with individual BNNSs. The agglomeration of BNNSs within nanocomposites usually occurs after a threshold loading amount of BNNSs. Such agglomerations can be reduced by increasing the compatibility of BNNSs with polymer matrix through functionalization. For instance, the attachment of hydrophilic functional groups to the surface of BNNSs is likely to yield uniform dispersion of BNNSs into the hydrophilic type of polymer matrices (e.g., polyacrylamide, poly(ethylene glycol) derivatives) owing to hydrophilic-hydrophilic interaction and vice versa.

Given the mass barrier properties of 2D nanosheets, the high surface area of BNNSs has the potential to reduce the oxygen and decomposed volatile gas flow within the nanocomposite matrix. Hence, reduced oxygen and decomposed gas flow promote the thermal stability of nanocomposites. On the other hand, the incorporation of BNNSs into polymer materials affects the optical properties of the corresponding composite materials. The main reason for the optical property changes is the light scattered by the BNNSs. The nanosheet size and agglomerates of BNNSs, and the defects formed within the nanocomposites are the source of such light scattering. Different strategies are developed to minimize the light scattering such as distributing the BNNSs uniformly within matrix, developing stronger interphase, and downsizing the BN nanosheet sizes. Such approaches require good control of downsizing BNNSs as well as modification of BNNSs. The surface modification includes functionalizing BNNSs with appropriate functional molecules in order to increase the miscibility of nanosheets into polymer matrix and form chemical bonding between nanocomposite constituents. The other optical properties such as UV and IR-wavelength blocking also require 
good control of the above-mentioned factors to develop the desired properties into polymer nanocomposites.

In the future, attention should be given to the following: (i) to study the polymer-BNNS interphases in more details in terms of their molecular structure and chemistry, and develop structure-property relationships between interphase and properties such as stress transfer, polymer chain dynamics, UV-light transparency, and thermal resistance, (ii) to investigate the mechanisms leading to the aggregation of BN nanosheets depending on the polymer chemistry, functionalized interfaces, and the mitigation strategies, (iii) to explore strategies that can increase the chemical reactivity of BNNSs with the polymer matrix, and (iv) to develop low-cost scalable synthesis of high-quality $\mathrm{BN}$ nanosheets and polymer nanocomposites in order to make them appealing for industrial applications. It should be mentioned that the industrial-scale exfoliation of BN nanosheets is still very costly. This is because of the low yield (5-30\%) of BNNSs exfoliation resulting from the firm "lip-lip" interaction between B-N bonds ${ }^{228}$. It is important to explore environment friendly and facile routes for low-cost, large-scale synthesis of BN nanosheets.

\section{DATA AVAILABILITY}

Data sharing is not applicable to this article as no new data were created or analyzed in this study.

Received: 14 September 2020; Accepted: 31 March 2021; Published online: 17 May 2021

\section{REFERENCES}

1. Naskar, A. K., Keum, J. K. \& Boeman, R. G. Polymer matrix nanocomposites for automotive structural components. Nat. Nanotechnol. 11, 1026-1030 (2016).

2. Garcés, J. M., Moll, D. J., Bicerano, J., Fibiger, R. \& McLeod, D. G. Polymeric nanocomposites for automotive applications. Adv. Mater. 12, 1835-1839 (2000).

3. Barua, L., Zou, B. \& Zhou, Y. Machine learning for international freight transportation management: a comprehensive review. Res. Transp. Bus. Manag. 34, 100453 (2020).

4. Hussain, Farzana \& Hojjati, M. Polymer-matrix nanocomposites, processing, manufacturing, and application: an overview. J. Compos. Mater. 40, 467-471 (2006).

5. Barua, L., Zou, B., Noruzoliaee, M. \& Derrible, S. A gradient boosting approach to understanding airport runway and taxiway pavement deterioration. Int. J. Pavement Eng. 1-15 (2020).

6. Ciofani, G., Raffa, V., Menciassi, A. \& Cuschieri, A. Cytocompatibility, interactions, and uptake of polyethyleneimine-coated boron nitride nanotubes by living cells: Confirmation of their potential for biomedical applications. Biotechnol. Bioeng. 101, 850-858 (2008).

7. Chen, $X$. et al. Boron nitride nanotubes are noncytotoxic and can be functionalized for interaction with proteins and cells. J. Am. Chem. Soc. 131, 890-891 (2009).

8. Horváth, L. et al. In vitro investigation of the cellular toxicity of boron nitride nanotubes. ACS Nano 5, 3800-3810 (2011).

9. Lei, W. et al. Oxygen-doped boron nitride nanosheets with excellent performance in hydrogen storage. Nano Energy 6, 219-224 (2014).

10. Li, Q. et al. Sandwich-structured polymer nanocomposites with high energy density and great charge-discharge efficiency at elevated temperatures. Proc. Natl Acad. Sci. USA 113, 9995-10000 (2016).

11. Yu, Y.-X. A dispersion-corrected DFT study on adsorption of battery active materials anthraquinone and its derivatives on monolayer graphene and $\mathrm{h}-\mathrm{BN}$. J. Mater. Chem. A 2, 8910-8917 (2014).

12. Jang, S. K., Youn, J., Song, Y. J. \& Lee, S. Synthesis and characterization of hexagonal boron nitride as a gate dielectric. Sci. Rep. 6, 30449 (2016).

13. Bai, S. \& Shen, X. Graphene-inorganic nanocomposites. RSC Adv. 2, 64-98 (2012).

14. Wang, D. et al. Surface modification of graphene with layered molybdenum disulfide and their synergistic reinforcement on reducing fire hazards of epoxy resins. Ind. Eng. Chem. Res. 52, 17882-17890 (2013).

15. Qi, B. et al. Enhanced thermal and mechanical properties of epoxy composites by mixing thermotropic liquid crystalline epoxy grafted graphene oxide. Express Polym. Lett. 8, 467-479 (2014).

16. Domun, N. et al. Improving the fracture toughness properties of epoxy using graphene nanoplatelets at low filler content. Nanocomposites 3, 85-96 (2017).
17. Chandrasekaran, S. et al. Fracture toughness and failure mechanism of graphene based epoxy composites. Compos. Sci. Technol. 97, 90-99 (2014).

18. Qi, B. et al. Mechanical and thermal properties of epoxy composites containing graphene oxide and liquid crystalline epoxy. Fibers Polym. 15, 326-333 (2014).

19. Ramos-Galicia, L. et al. Improved performance of an epoxy matrix as a result of combining graphene oxide and reduced graphene. Int. J. Polym. Sci. 2013, 1-7 (2013).

20. Boldrin, L., Scarpa, F., Chowdhury, R. \& Adhikari, S. Effective mechanical properties of hexagonal boron nitride nanosheets. Nanotechnology 22, 505702 (2011).

21. Li, L. H., Cervenka, J., Watanabe, K., Taniguchi, T. \& Chen, Y. Strong oxidation resistance of atomically thin boron nitride nanosheets. ACS Nano 8, 1457-1462 (2014).

22. Verma, A., Parashar, A. \& Packirisamy, M. Tailoring the failure morphology of $2 D$ bicrystalline graphene oxide. J. Appl. Phys. 124, 015102 (2018).

23. Paszkowicz, W., Pelka, J. B., Knapp, M., Szyszko, T. \& Podsiadlo, S. Lattice parameters and anisotropic thermal expansion of hexagonal boron nitride in the 10-297.5 K temperature range. Appl. Phys. A Mater. Sci. Process. 75, 431-435 (2002).

24. Meng, W., Huang, Y., Fu, Y., Wang, Z. \& Zhi, C. Polymer composites of boron nitride nanotubes and nanosheets. J. Mater. Chem. C. 2, 10049-10061 (2014).

25. Weng, Q., Wang, X., Wang, X., Bando, Y. \& Golberg, D. Functionalized hexagonal boron nitride nanomaterials: emerging properties and applications. Chem. Soc. Rev. 45, 3989-4012 (2016).

26. Ling, Z. et al. Flexible and conductive $M X e n e$ films and nanocomposites with high capacitance. Proc. Natl. Acad. Sci. 111, 16676-16681 (2014).

27. Li, Y. Q., Yu, T., Yang, T. Y., Zheng, L. X. \& Liao, K. Bio-Inspired nacre-like composite films based on graphene with superior mechanical, electrical, and biocompatible properties. Adv. Mater. 24, 3426-3431 (2012).

28. Zhang, M., Huang, L., Chen, J., Li, C. \& Shi, G. Ultratough, ultrastrong, and highly conductive graphene films with arbitrary sizes. Adv. Mater. 26, 7588-7592 (2014).

29. Narayanan, S. et al. On the structure and chemistry of iron oxide cores in human heart and human spleen ferritins using graphene liquid cell electron microscopy. Nanoscale 11, 16868-16878 (2019).

30. Wen, Y. et al. Direct growth of tungsten disulfide (WS2) on gallium nitride (GaN) and the photovoltaic characteristics of the heterojunctions. Semicond. Sci. Technol. 36, 025016 (2020).

31. Dean, C. R. et al. Boron nitride substrates for high-quality graphene electronics. Nat. Nanotechnol. 5, 722-726 (2010).

32. Ren, X. et al. One-pot polymer conjugation on carbon nanotubes through simultaneous $\mathrm{p}$ e $\mathrm{p}$ stacking and the Biginelli reaction. Polymer 64, 210-215 (2015).

33. Li, K., Liu, J., Huang, Y., Bu, F. \& Xu, Y. Integration of ultrathin graphene/polyaniline composite nanosheets with a robust $3 D$ graphene framework for highly flexible all-solid-state supercapacitors with superior energy density and exceptional cycling stability. J. Mater. Chem. A 5, 5466-5474 (2017).

34. Roenbeck, M. R. et al. Molecular-level engineering of adhesion in carbon nanomaterial interfaces. Nano Lett. 15, 4504-4516 (2015).

35. Chen, X. et al. Quantitative nanomechanical characterization of the van der Waals interfaces between carbon nanotubes and epoxy. Carbon N. Y. 82 214-228 (2015).

36. Chen, X., Zheng, M., Park, C. \& Ke, C. Direct measurements of the mechanical strength of carbon nanotube-poly(methyl methacrylate) interfaces. Small $\mathbf{9}$, 3345-51 (2013).

37. Chen, X. et al. Mechanical strength of boron nitride nanotube-polymer interfaces. Appl. Phys. Lett. 107, 253105 (2015).

38. Rouhi, S. Molecular dynamics simulation of the adsorption of polymer chains on CNTs, BNNTs and GaNNTs. Fibers Polym. 17, 333-342 (2016).

39. Wang, X., Zhi, C., Weng, Q., Bando, Y. \& Golberg, D. Boron nitride nanosheets: novel syntheses and applications in polymeric composites. J. Phys. Conf. Ser. 471, 012003 (2013)

40. Yu, C., Zhang, J., Tian, W., Fan, X. \& Yao, Y. Polymer composites based on hexagonal boron nitride and their application in thermally conductive composites. RSC Adv. 8, 21948-21967 (2018).

41. Verma, A., Parashar, A. \& Packirisamy, M. Atomistic modeling of graphene/ hexagonal boron nitride polymer nanocomposites: a review. Wiley Interdiscip. Rev. Comput. Mol. Sci. 8, https://doi.org/10.1002/wcms.1346 (2018).

42. Zhi, C. Y., Bando, Y., Tang, C. C., Huang, Q. \& Golberg, D. Boron nitride nanotubes: functionalization and composites. J. Mater. Chem. 18, 3900-3908 (2008).

43. Guerra, V., Wan, C. \& McNally, T. Thermal conductivity of 2D nano-structured boron nitride (BN) and its composites with polymers. Prog. Mater. Sci. 100, 170-186 (2019).

44. Zheng, Z., Cox, M. C. \& Li, B. Surface modification of hexagonal boron nitride nanomaterials: a review. J. Mater. Sci. 53, 66-99 (2018). 
45. Pakdel, A., Wang, X., Bando, Y. \& Golberg, D. Nonwetting 'white graphene' films. Acta Mater. 61, 1266-1273 (2013).

46. Li, J., Chen, J., Zhu, M., Song, H. \& Zhang, H. Interfacial characteristics of boron nitride nanosheet/epoxy resin nanocomposites: a molecular dynamics simulation. Appl. Sci. 9, 2832 (2019).

47. Roy, S., Petrova, R. S. \& Mitra, S. Effect of carbon nanotube (CNT) functionalization in epoxy-CNT composites. Nanotechnol. Rev. 7, 475-485 (2018).

48. Eitan, A., Jiang, K., Dukes, D., Andrews, R. \& Schadler, L. S. Surface modification of multiwalled carbon nanotubes: toward the tailoring of the interface in polymer composites. Chem. Mater. 15, 3198-3201 (2003).

49. $\mathrm{Yu}, \mathrm{J}$. et al. Interfacial modification of boron nitride nanoplatelets for epoxy composites with improved thermal properties. Polymer 53, 471-480 (2012).

50. Guo, Y., Ruan, K., Shi, X., Yang, X. \& Gu, J. Factors affecting thermal conductivities of the polymers and polymer composites: a review. Compos. Sci. Technol. 193, 108134 (2020).

51. Radhakrishnan, S. et al. Fluorinated h-BN as a magnetic semiconductor. Sci. Adv. 3, e1700842 (2017)

52. Yang, H. et al. Influence of doped nitrogen and vacancy defects on the thermal conductivity of graphene nanoribbons. J. Mol. Model. 19, 4781-4788 (2013).

53. Abdelmouleh, M., Boufi, S., Belgacem, M. N. \& Dufresne, A. Short natural-fibre reinforced polyethylene and natural rubber composites: effect of silane coupling agents and fibres loading. Compos. Sci. Technol. 67, 1627-1639 (2007).

54. Rong, M. Z., Zhang, M. Q. \& Ruan, W. H. Surface modification of nanoscale fillers for improving properties of polymer nanocomposites: a review. Mater. Sci. Technol. 22, 787-796 (2006).

55. Rasul, M. G. et al. Polyethylene-BN nanosheets nanocomposites with enhanced thermal and mechanical properties. Compos. Sci. Technol. 204, 108631 (2021).

56. Lipatov, Y. S. Interfaces in polymer-polymer composites. In: Controlled Interphases in Composite Materials (eds. Ishida H.) 599-611 (Springer, 1990).

57. Nikkhah, S. J., Moghbeli, M. R. \& Hashemianzadeh, S. M. Interfacial adhesion between functionalized polyethylene surface and graphene via molecular dynamic simulation. J. Mol. Model. 21, 121 (2015).

58. Aluko, O., Gowtham, S. \& Odegard, G. M. Multiscale modeling and analysis of graphene nanoplatelet/carbon fi ber / epoxy hybrid composite. Compos. Part $B$ 131, 82-90 (2017).

59. Hadden, C. M. et al. Molecular modeling of EPON-862 / graphite composites: Interfacial characteristics for multiple crosslink densities. Compos. Sci. Technol. 76, 92-99 (2013).

60. Mansfield, K. F. \& Theodorou, D. N. Atomistic simulation of a glassy polymer/ graphite interface. Macromolecules 24, 4295-4309 (1991).

61. Hadden, C. M. et al. Mechanical properties of graphene nanoplatelet/carbon $\mathrm{fi}$ ber / epoxy hybrid composites: Multiscale modeling and experiments. Carbon N. Y. 95, 100-112 (2015).

62. Li, C., Browning, A. R., Christensen, S. \& Strachan, A. Composites: part A atomistic simulations on multilayer graphene reinforced epoxy composites. Compos. Part A 43, 1293-1300 (2012).

63. Zhang, Y., Zhuang, X., Muthu, J., Mabrouki, T. \& Fontaine, M. Composites: Part B Load transfer of graphene/carbon nanotube/polyethylene hybrid nanocomposite by molecular dynamics simulation. Compos. Part B 63, 27-33 (2014).

64. Wan, C. \& Chen, B. Reinforcement and interphase of polymer/graphene oxide nanocomposites. J. Mater. Chem. 22, 3637-3646 (2012).

65. Kovalev, A., Shulha, H., Lemieux, M., Myshkin, N. \& Tsukruk, V. V. Nanomechanical probing of layered nanoscale polymer films with atomic force microscopy. J. Mater. Res. 19, 716-728 (2004).

66. Netravali, Anil N., and K. L. Mittal. (eds) Interface/interphase in polymer nanocomposites. (John Wiley \& Sons, 2016).

67. Wang, X. et al. High-yield boron nitride nanosheets from 'chemical blowing': towards practical applications in polymer composites. J. Phys. Condens. Matter 24, 31 (2012)

68. Wang, X. et al. Large-surface-area BN nanosheets and their utilization in polymeric composites with improved thermal and dielectric properties. Nanoscale Res. Lett. 7, 1-7 (2012).

69. Nautiyal, P., Gupta, A., Seal, S., Boesl, B. \& Agarwal, A. Reactive wetting and filling of boron nitride nanotubes by molten aluminum during equilibrium solidification. Acta Mater. 126, 124-131 (2017).

70. van Engers, C. D. et al. Direct measurement of the surface energy of graphene. Nano Lett. 17, 3815-3821 (2017).

71. Rathod, N. \& Hatzikiriakos, S. G. The effect of surface energy of boron nitride on polymer processability. Polym. Eng. Sci. 44, 1543-1550 (2004).

72. $\mathrm{Wu}, \mathrm{H}$. Multifunctional polymer composites containing inorganic nanoparticles and novel low- cost carbonaceous fillers. (lowa State University, 2014).

73. Luo, H. et al. Interface design for high energy density polymer nanocomposites. Chem. Soc. Rev. 48, 4424-4465 (2019).

74. Naguib, Y. W. \& Cui, Z. Nanomedicine: the promise and challenges in cancer chemotherapy. Adv. Exp. Med. Biol. 811, 207-33 (2014).
75. Qiao, R., Deng, H., Putz, K. W. \& Brinson, L. C. Effect of particle agglomeration and interphase on the glass transition temperature of polymer nanocomposites. $J$. Polym. Sci. Part B Polym. Phys. 49, 740-748 (2011).

76. Gojny, F. H. et al. Evaluation and identification of electrical and thermal conduction mechanisms in carbon nanotube/epoxy composites. Polymer 47, 2036-2045 (2006).

77. McNamara, A. J., Joshi, Y. \& Zhang, Z. M. Characterization of nanostructured thermal interface materials-a review. Int. J. Therm. Sci. 62, 2-11 (2012).

78. Shin, H., Yang, S., Choi, J., Chang, S. \& Cho, M. Effect of interphase percolation on mechanical behavior of nanoparticle-reinforced polymer nanocomposite with filler agglomeration: a multiscale approach. Chem. Phys. Lett. 635, 80-85 (2015).

79. Zare, Y., Rhee, K. Y. \& Hui, D. Influences of nanoparticles aggregation/agglomeration on the interfacial/interphase and tensile properties of nanocomposites. Compos. Part B Eng. 122, 41-46 (2017).

80. Zare, Y. Study of nanoparticles aggregation/agglomeration in polymer particulate nanocomposites by mechanical properties. Compos. Part A Appl. Sci. Manuf. 84, 158-164 (2016).

81. Min, Y. J., Kang, K. H. \& Kim, D. E. Development of polyimide films reinforced with boron nitride and boron nitride nanosheets for transparent flexible device applications. Nano Res. 11, 2366-2378 (2018).

82. Lin, Y. et al. Aqueous dispersions of few-layered and monolayered hexagonal boron nitride nanosheets from sonication-assisted hydrolysis: Critical role of water. J. Phys. Chem. C. 115, 2679-2685 (2011).

83. Jordan, J., Jacob, K. I., Tannenbaum, R., Sharaf, M. A. \& Jasiuk, I. Experimental trends in polymer nanocomposites - a review. Mater. Sci. Eng. A 393, 1-11 (2005).

84. Compton, O. C. et al. Additive-free hydrogelation of graphene oxide by ultrasonication. Carbon N. Y. 50, 3399-3406 (2012).

85. Honda, S., Yamamoto, T. \& Tezuka, Y. Supporting information topology-directed control on thermal stability: micelles formed from linear and cyclized. Solutions 132, 10251-3 (2010).

86. Zhong, M., Wang, R., Kawamoto, K., Olsen, B. D. \& Johnson, J. A. Supplementary materials for quantifying the impact of molecular defects on polymer network elasticity. Science 353, 1264 (2016).

87. Abadi, M., Serag, M. F. \& Habuchi, S. Entangled polymer dynamics beyond reptation. Nat. Commun. 9, 5098 (2018).

88. Doi, M. \& Edwards, S. F. The Theory of Polymer Dynamics. (Oxford University Press, 1986).

89. Peña-Bahamonde, J. et al. Effect of polysulfone brush functionalization on thermo-mechanical properties of melt extruded graphene/polysulfone nanocomposites. Carbon 151, 84-93 (2019).

90. Gonzalez-Ortiz, D., Salameh, C., Bechelany, M. \& Miele, P. Nanostructured boron nitride-based materials: synthesis and applications. Mater. Today Adv. 8, 100107 (2020).

91. Du, X. S., Xiao, M. \& Meng, Y. Z. Synthesis and characterization of polyaniline/ graphite conducting nanocomposites. J. Polym. Sci. Part B Polym. Phys. 42, 1972-1978 (2004).

92. Kiran, M. S. R. N., Raidongia, K., Ramamurty, U. \& Rao, C. N. R. Improved mechanical properties of polymer nanocomposites incorporating graphene-like BN: dependence on the number of BN layers. Scr. Mater. 64, 592-595 (2011).

93. Donnay, M., Tzavalas, S. \& Logakis, E. Boron nitride filled epoxy with improved thermal conductivity and dielectric breakdown strength. Compos. Sci. Technol. 110, 152-158 (2015).

94. Yamazaki, S. et al. Effect of entanglement on nucleation rate of polyethylene. Polym. J. 33, 906-908 (2003).

95. Yunsheng, X. \& Chung, D. D. L. Increasing the thermal conductivity of BN and AIN in epoxy-matrix composites by particle surface treatments. 7, 243-256 (2000).

96. Takahashi, S., Imai, Y., Kan, A., Hotta, Y. \& Ogawa, H. Dielectric and thermal properties of isotactic polypropylene/hexagonal boron nitride composites for high-frequency applications. J. Alloy. Compd. 615, 141-145 (2014).

97. Çamurlu, H. E., Akarsu, E., Arslan, O. \& Mathur, S. Nanocomposite glass coatings containing hexagonal boron nitride nanoparticles. Ceram. Int. 42, 8856-8862 (2016).

98. Cheng, J. J., Alvarado-Contreras, J. A., Polak, M. A. \& Penlidis, A. Chain entanglements and mechanical behavior of high density polyethylene. J. Eng. Mater. Technol. 132, 011016 (2010).

99. Kim, H., Miura, Y. \& MacOsko, C. W. Graphene/polyurethane nanocomposites for improved gas barrier and electrical conductivity. Chem. Mater. 22, 3441-3450 (2010).

100. Cho, D., Lee, S., Yang, G., Fukushima, H. \& Drzal, L. T. Dynamic mechanical and thermal properties of phenylethynyl-terminated polyimide composites reinforced with expanded graphite nanoplatelets. Macromol. Mater. Eng. 290, 179-187 (2005).

101. Lin, Z. et al. Magnetic alignment of hBN platelets in polymer matrix toward high performance anisotropic polymer composite for electronic encapsulation. Appl. Mater. Interfaces 5, 7633-7640 (2013). 
102. Jan, R. et al. Enhancing the mechanical properties of BN nanosheet-polymer composites by uniaxial drawing. Nanoscale 6, 4889-4895 (2014).

103. Coleman, J. N., Khan, U. \& Gun'ko, Y. K. Mechanical reinforcement of polymers using carbon nanotubes. Adv. Mater. 18, 689-706 (2006).

104. Kelly, A. \& Macmillan, N. H. Strong solids. (Clarendon Press, 1986).

105. Wang, Y., Shi, Z. \& Yin, J. Boron nitirde nanosheets: large-scale exfoliation in methanesulfonic acid and their composites with polybenzimidazole. J. Mater. Chem. 21, 11371-11377 (2011).

106. Hu, C., Liao, X., Qin, Q. H. \& Wang, G. The fabrication and characterization of high density polyethylene composites reinforced by carbon nanotube coated carbon fibers. Compos. Part A Appl. Sci. Manuf. 121, 149-156 (2019).

107. Chaurasia, A., Verma, A., Parashar, A. \& Mulik, R. S. Experimental and computational studies to analyze the effect of $h-B N$ nanosheets on mechanical behavior of h-BN/polyethylene nanocomposites. J. Phys. Chem. C. 123, 20059-20070 (2019).

108. Fang, H., Bai, S. L. \& Wong, C. P. Thermal, mechanical and dielectric properties of flexible BN foam and BN nanosheets reinforced polymer composites for electronic packaging application. Compos. Part A Appl. Sci. Manuf. 100, 71-80 (2017).

109. Wang, X. Bin et al. Biomass-directed synthesis of $20 \mathrm{~g}$ high-quality boron nitride nanosheets for thermoconductive polymeric composites. ACS Nano $\mathbf{8}$, 9081-9088 (2014).

110. Li, S., Yang, T., Zou, H., Liang, M. \& Chen, Y. Enhancement in thermal conductivity and mechanical properties via large-scale fabrication of boron nitride nanosheets. High. Perform. Polym. 29, 315-327 (2017).

111. $\mathrm{Hu}, \mathrm{G}$. et al. Functional inks and printing of two-dimensional materials. Chem. Soc. Rev. 47, 3265-3300 (2018).

112. Xue, S. et al. Fabrication of poly(acrylic acid)/boron nitride composite hydrogels with excellent mechanical properties and rapid self-healing through hierarchically physical interactions. Nanoscale Res. Lett. 13, 393 (2018),

113. Tajaddod, N., Song, K., Green, E. C., Zhang, Y. \& Minus, M. L. Exfoliation of boron nitride platelets by enhanced interfacial interaction with polyethylene. Macromol. Mater. Eng. 301, 315-327 (2016).

114. Ji, Z. et al. Mechanical and tribological properties of nanocomposites incorporated with two-dimensional materials. Friction 8, 813-846 (2020).

115. Zhou, K., Gao, R., Gui, Z. \& Hu, Y. The effective reinforcements of functionalized MoS2 nanosheets in polymer hybrid composites by sol-gel technique. Compos. Part A Appl. Sci. Manuf. 94, 1-9 (2017).

116. Papageorgiou, D. G., Li, Z., Liu, M., Kinloch, I. A. \& Young, R. J. Mechanisms of mechanical reinforcement by graphene and carbon nanotubes in polymer nanocomposites. Nanoscale 12, 2228-2267 (2020)

117. Burger, N. et al. Review of thermal conductivity in composites: mechanisms, parameters and theory. Prog. Polym. Sci. 61, 1-28 (2016).

118. Konatham, D. \& Striolo, A. Thermal boundary resistance at the graphene-oil interface. Appl. Phys. Lett. 95, 93-96 (2009).

119. Wang, Y., Yang, C., Pei, Q. X. \& Zhang, Y. Some aspects of thermal transport across the interface between graphene and epoxy in nanocomposites. ACS Appl. Mater. Interfaces 8, 8272-8279 (2016).

120. Wang, Y., Yang, C., Mai, Y. W. \& Zhang, Y. Effect of non-covalent functionalisation on thermal and mechanical properties of graphene-polymer nanocomposites. Carbon 102, 311-318 (2016).

121. Konatham, D., Bui, K. N. D., Papavassiliou, D. V. \& Striolo, A. Simulation insights into thermally conductive graphene-based nanocomposites. Mol. Phys. 109, 97-111 (2011).

122. Xiao, F. et al. Edge-hydroxylated boron nitride nanosheets as an effective additive to improve the thermal response of hydrogels. Adv. Mater. 27, 7196-7203 (2015)

123. Liu, J., Zhang, H., Zhang, Z. \& Fabrication, H. High thermal conductivity composite with shear- induced alligned boron nitride nanosheets. in 21st International Conference on Composite Materials (ed. Shanyi Du, J. L.) (Chinese Society for Composite Materials, 2017).

124. Zhu, Z. et al. Enhanced thermal conductivity of polyurethane composites via engineering small/large sizes interconnected boron nitride nanosheets. Compos. Sci. Technol. 170, 93-100 (2019).

125. Wang, X. \& Wu, P. Preparation of highly thermally conductive polymer composite at low filler content via a self-assembly process between polystyrene microspheres and boron nitride nanosheets. ACS Appl. Mater. Interfaces 9, 19934-19944 (2017).

126. Wu, K. et al. Achieving a collapsible, strong, and highly thermally conductive film based on oriented functionalized boron nitride nanosheets and cellulose nanofiber. ACS Appl. Mater. Interfaces 9, 30035-30045 (2017).

127. Lin, Z. et al. Magnetic alignment of hexagonal boron nitride platelets in polymer matrix: toward high performance anisotropic polymer composites for electronic encapsulation. ACS Appl. Mater. Interfaces 5, 7633-7640 (2013).
128. Yuan, C. et al. Thermal conductivity of polymer-based composites with magnetic aligned hexagonal boron nitride platelets. ACS Appl. Mater. Interfaces 7 , 13000-13006 (2015).

129. Yuan, C., Xie, B., Huang, M., Wu, R. \& Luo, X. Thermal conductivity enhancement of platelets aligned composites with volume fraction from $10 \%$ to $20 \%$. Int. J. Heat. Mass Transf. 94, 20-28 (2016).

130. Chen, J. et al. Vertically aligned and interconnected boron nitride nanosheets for advanced flexible nanocomposite thermal interface materials. ACS Appl. Mater. Interfaces 9, 30909-30917 (2017).

131. Morishita, T. \& Okamoto, H. Facile exfoliation and noncovalent superacid functionalization of boron nitride nanosheets and their use for highly thermally conductive and electrically insulating polymer nanocomposites. ACS Appl. Mater. Interfaces 8, 27064-27073 (2016).

132. Zhu, H. et al. Highly thermally conductive papers with percolative layered boron nitride nanosheets. ACS Nano 8, 3606-3613 (2014).

133. Hou, J. et al. Preparation and characterization of surface modified boron nitride epoxy composites with enhanced thermal conductivity. RSC Adv. 4, 44282-44290 (2014)

134. Yu, J., Mo, H. \& Jiang, P. Polymer/boron nitride nanosheet composite with high thermal conductivity and sufficient dielectric strength. Polym. Adv. Technol. 26, 514-520 (2015).

135. Cui, X. et al. Thermal conductive and mechanical properties of polymeric composites based on solution-exfoliated boron nitride and graphene nanosheets: a morphology-promoted synergistic effect. ACS Appl. Mater. Interfaces 7 19068-19075 (2015)

136. Haggenmueller, R., Guthy, C., Lukes, J. R., Fischer, J. E. \& Winey, K. I. Single wall carbon nanotube/polyethylene nanocomposites: thermal and electrical conductivity. Macromolecules 40, 2417-2421 (2007).

137. Shtein, M., Nadiv, R., Buzaglo, M., Kahil, K. \& Regev, O. Thermally conductive graphene-polymer composites: size, percolation, and synergy effects. Chem. Mater. 27, 2100-2106 (2015).

138. Xie, B. H., Huang, X. \& Zhang, G. J. High thermal conductive polyvinyl alcohol composites with hexagonal boron nitride microplatelets as fillers. Compos. Sci. Technol. 85, 98-103 (2013)

139. Tanimoto, M., Yamagata, T., Miyata, K. \& Ando, S. Anisotropic thermal diffusivity of hexagonal boron nitride-filled polyimide films: effects of filler particle size, aggregation, orientation, and polymer chain rigidity. ACS Appl. Mater. Interfaces 5, 4374-4382 (2013).

140. Song, W. L. et al. Polymer/boron nitride nanocomposite materials for superior thermal transport performance. Angew. Chem. Int. Ed. 51, 6498-6501 (2012).

141. Kim, K. \& Kim, J. Vertical filler alignment of boron nitride/epoxy composite for thermal conductivity enhancement via external magnetic field. Int. J. Therm. Sci. 100, 29-36 (2016).

142. Cho, H. B. et al. Insulating polymer nanocomposites with high-thermalconduction routes via linear densely packed boron nitride nanosheets. Compos. Sci. Technol. 129, 205-213 (2016).

143. Zhai, S., Zhang, P., Xian, Y., Zeng, J. \& Shi, B. Effective thermal conductivity of polymer composites: theoretical models and simulation models. Int. J. Heat. Mass Transf. 117, 358-374 (2018).

144. Zhai, S., Zhang, P., Xian, Y., Yuan, P. \& Yang, D. Modelling and analysis of effective thermal conductivity for polymer composites with sheet-like nanoparticles. J. Mater. Sci. 54, 356-369 (2019).

145. He, J. et al. Fabrication of boron nitride nanosheet/polymer composites with tunable thermal insulating properties. N. J. Chem. 43, 4878-4885 (2019).

146. Zanetti, M., Camino, G., Thomann, R. \& Mülhaupt, R. Synthesis and thermal behaviour of layered silicate-EVA nanocomposites. Polymer 42, 4501-4507 (2001).

147. Alexandre, M. \& Dubois, P. Polymer-layered silicate nanocomposites: preparation, properties and uses of a new class of materials. Mater. Sci. Eng. R. Rep. 28, 1-63 (2000).

148. Zhu, J., Morgan, A. B., Lamelas, F. J. \& Wilkie, C. A. Fire properties of polystyreneclay nanocomposites. Chem. Mater. 13, 3774-3780 (2001).

149. Burnside, S. D. \& Giannelis, E. P. Synthesis and properties of new poly(Dimethylsiloxane) nanocomposites. Chem. Mater. 7, 1597-1600 (1995).

150. Gilman, J. W. Flammability and thermal stability studies of polymer layeredsilicate (clay) nanocomposites. Appl. Clay Sci. 15, 31-49 (1999).

151. Tidjani, A., Wald, O., Pohl, M. M., Hentschel, M. P. \& Schartel, B. Polypropylenegraft-maleic anhydride-nanocomposites: I - characterization and thermal stability of nanocomposites produced under nitrogen and in air. Polym. Degrad. Stab. 82, 133-140 (2003).

152. Nguyen, H. L. et al. Sustainable boron nitride nanosheet-reinforced cellulose nanofiber composite film with oxygen barrier without the cost of color and cytotoxicity. Polym. (Basel). 10, 1-11 (2018). 
153. Müller, K. et al. Review on the processing and properties of polymer nanocomposites and nanocoatings and their applications in the packaging, automotive and solar energy fields. Nanomaterials 7, 74 (2017).

154. Paul, D. R. \& Robeson, L. M. Polymer nanotechnology: nanocomposites. Polymer 49, 3187-3204 (2008).

155. Madakbaş, S., Çakmakçi, E. \& Kahraman, M. V. Preparation and thermal properties of polyacrylonitrile/hexagonal boron nitride composites. Thermochim. Acta 552, 1-4 (2013).

156. Isarn, I., Ramis, X., Ferrando, F. \& Serra, A. Thermoconductive thermosetting composites based on boron nitride fillers and thiol-epoxy matrices. Polymers (Basel). 10, 277 (2018).

157. Li, T. L. \& Hsu, S. L. C. Enhanced thermal conductivity of polyimide films via a hybrid of micro- and nano-sized boron nitride. J. Phys. Chem. B 114, 6825-6829 (2010).

158. Zhi, C. et al. Boron nitride nanotubes/polystyrene composites. J. Mater. Res. 21, 2794-2800 (2006).

159. Li, T.-L. \& Hsu, S. L.-C. Preparation and properties of thermally conductive photosensitive polyimide/boron nitride nanocomposites. Rawal Med. J. 43, 483-485 (2010).

160. Vyazovkin, S., Dranca, I., Fan, X. \& Advincula, R. Degradation and relaxation kinetics of polystyrene-clay nanocomposite prepared by surface initiated polymerization. J. Phys. Chem. B 108, 11672-11679 (2004).

161. Vyazovkin, S. \& Dranca, I. A DSC study of a- and $\beta$-relaxations in a PS-clay system. J. Phys. Chem. B 108, 11981-11987 (2004).

162. Costache, M. C., Wang, D., Heidecker, M. J., Manias, E. \& Wilkie, C. A. The thermal degradation of poly(methyl methacrylate) nanocomposites with montmorillonite, layered double hydroxides and carbon nanotubes. Polym. Adv. Technol. 17, 272-280 (2006).

163. Jang, B. N. \& Wilkie, C. A. The effects of clay on the thermal degradation behavior of poly(styrene-co-acrylonitirile). Polymer 46, 9702-9713 (2005).

164. Jang, B. N. \& Wilkie, C. A. The thermal degradation of polystyrene nanocomposite. Polymer 46, 2933-2942 (2005).

165. Jang, B. N. \& Wilkie, C. A. The effect of clay on the thermal degradation of polyamide 6 in polyamide 6/clay nanocomposites. Polymer 46, 3264-3274 (2005).

166. Jang, B. N., Costache, M. \& Wilkie, C. A. The relationship between thermal degradation behavior of polymer and the fire retardancy of polymer/clay nanocomposites. Polymer 46, 10678-10687 (2005).

167. Qiu, L., Chen, W. \& Qu, B. Morphology and thermal stabilization mechanism of LLDPE/MMT and LLDPE/LDH nanocomposites. Polymer 47, 922-930 (2006).

168. Gao, Y. Y., Hu, F. Y., Liu, J. \& Wang, Z.. Molecular dynamics simulation of the glass transition temperature of fullerene filled cis-1,4-polybutadiene nanocomposites. Chinese J. Polym. Sci. 36, 119-128 (2018)

169. Forrest, J. A., Dalnoki-Veress, K. \& Dutcher, J. R. Interface and chain confinement effects on the glass transition temperature of thin polymer films. Phys. Rev. E 56, 5705-5716 (1997).

170. Xie, S.-J., Qian, H.-J. \& Lu, Z.-Y. Hard and soft confinement effects on the glass transition of polymers confined to nanopores. Polymer 56, 545-552 (2015).

171. White, R. P., Price, C. C. \& Lipson, J. E. G. Effect of interfaces on the glass transition of supported and freestanding polymer thin films. Macromolecules 48, 4132-4141 (2015).

172. Talik, A. et al. The role of interfacial energy and specific interactions on the behavior of poly(propylene glycol) derivatives under 2D confinement. Macromolecules 51, 4840-4852 (2018).

173. Chantawansri, T. L., Yeh, I. C. \& Hsieh, A. J. Investigating the glass transition temperature at the atom-level in select model polyamides: a molecular dynamics study. Polymer 81, 50-61 (2015).

174. Khabaz, F. \& Khare, R. Glass transition and molecular mobility in styrenebutadiene rubber modified asphalt. J. Phys. Chem. B 119, 14261-14269 (2015).

175. Soldera, A. \& Metatla, N. Glass transition of polymers: atomistic simulation versus experiments. Phys. Rev. E Stat. Nonlinear, Soft Matter Phys. 74, 1-6 (2006).

176. Harmandaris, V. A., Floudas, G. \& Kremer, K. Temperature and pressure dependence of polystyrene dynamics through molecular dynamics simulations and experiments. Macromolecules 44, 393-402 (2011).

177. Wu, R., Zhang, X., Ji, Q., Kong, B. \& Yang, X. Conformational transition behavior of amorphous polyethylene across the glass transition temperature. J. Phys. Chem. B 113, 9077-9083 (2009).

178. Zhang, J., Yan, J., Uitenham, L. \& Lou, J. Study of the molecular weight dependence of glass transition temperature for amorphous poly(l-lactide) by molecular dynamics simulation. Polymer 48, 339-344 (2009).

179. Starr, F. W., Douglas, J. F., Meng, D. \& Kumar, S. K. Bound layers "Cloak" nanoparticles in strongly interacting polymer nanocomposites. ACS Nano 10, 10960-10965 (2016).

180. Cheng, S. et al. Big effect of small nanoparticles: a shift in paradigm for polymer nanocomposites. ACS Nano 11, 752-759 (2017).
181. Smith, G. D., Bedrov, D. \& Borodin, O. Structural relaxation and dynamic heterogeneity in a polymer melt at attractive surfaces. Phys. Rev. Lett. 90, 4 (2003).

182. Torres, J. A., Nealey, P. F. \& De Pablo, J. J. Molecular simulation of ultrathin polymeric films near the glass transition. Phys. Rev. Lett. 85, 3221-3224 (2000).

183. Zhi, C., Bando, Y., Tang, C., Kuwahara, H. \& Golberg, D. Large-scale fabrication of boron nitride nanosheets and their utilization in polymeric composites with improved thermal and mechanical properties. Adv. Mater. 21, 2889-2893 (2009).

184. Li, Y., Wang, S. \& Wang, Q. Molecular dynamics simulations of thermal properties of polymer composites enhanced by cross-linked graphene sheets. Acta Mech. Solid. Sin. 31, 673-682 (2018).

185. Lu, H., Yao, Y., Huang, W. M., Leng, J. \& Hui, D. Significantly improving infrared light-induced shape recovery behavior of shape memory polymeric nanocomposite via a synergistic effect of carbon nanotube and boron nitride. Compos. Part B Eng. 62, 256-261 (2014).

186. Cakmakc, E., Kocyigit, C., Cakır, S., Durmus, A. \& Kahraman, M. V. Preparation and characterization of thermally conductive thermoplastic polyurethane/h-BN nanocomposites. Polym. Polym. Compos. 16, 101-113 (2008).

187. Yasaei, P. Thermal transport and power dissipation in two-dimensional (2D) materials and interfaces. ProQuest Diss. Theses 155 (2017).

188. Li, A., Zhang, C. \& Zhang, Y. F. Thermal conductivity of graphene-polymer composites: mechanisms, properties, and applications. Polym. (Basel). 9, 1-17 (2017).

189. Pielichowska, K., Szatkowski, P., Zambrzycki, M. \& Macherzynska, B. Polyurethane/graphene nanocomposites as phase change materials for thermal energy storage. in 2015 IEEE 15th International Conference on Nanotechnology (IEEE-NANO) 105-108 (IEEE, 2015). https://doi.org/10.1109/NANO.2015.7388808.

190. Cheng, M. et al. Direct ink writing of polymer composite electrolytes with enhanced thermal conductivities. Adv. Funct. Mater. 31, 2006683 (2021).

191. Melorose, J., Perroy, R. \& Careas, S. Dielectric Polymer. Statewide Agricultural Land Use Baseline 2015 1, (2015).

192. Tanaka, T., Montanari, G. C. \& Mülhaupt, R. Polymer nanocomposites as dielectrics and electrical insulation- perspectives for processing technologies, material characterization and future applications. IEEE Trans. Dielectr. Electr. Insul. 11, 763-784 (2004)

193. Johnson, R. W., Evans, J. L., Jacobsen, P., Thompson, J. R. \& Christopher, M. The changing automotive environment: high-temperature electronics. IEEE Trans. Electron. Packag. Manuf. 27, 164-176 (2004).

194. Li, Q. et al. Solution-processed ferroelectric terpolymer nanocomposites with high breakdown strength and energy density utilizing boron nitride nanosheets. Energy Environ. Sci. 8, 922-931 (2015).

195. Tsekmes, I. A., Kochetov, R., Morshuis, P. H. F. \& Smit, J. J. DC breakdown strength of epoxy-boron nitride nanocomposites: trend and reproducibility. in 2015 IEEE Conference on Electrical Insulation and Dielectric Phenomena (CEIDP) 479-482 (2015). https://doi.org/10.1109/CEIDP.2015.7352014.

196. Wang, Z., lizuka, T., Kozako, M., Ohki, Y. \& Tanaka, T. Development of epoxy/BN composites with high thermal conductivity and sufficient dielectric breakdown strength partl - sample preparations and thermal conductivity. IEEE Trans. Dielectr. Electr. Insul. 18, 1963-1972 (2011).

197. Xing, L. et al. Self-healable polymer nanocomposites capable of simultaneously recovering multiple functionalities. Adv. Funct. Mater. 26, 3524-3531 (2016).

198. Chen, J., Huang, X., Sun, B. \& Jiang, P. Highly thermally conductive yet electrically insulating polymer/boron nitride nanosheets nanocomposite films for improved thermal management capability. ACS Nano 13, 337-345 (2019).

199. Shim, J. et al. 2D boron nitride nanoflakes as a multifunctional additive in gel polymer electrolytes for safe, long cycle life and high rate lithium metal batteries. Energy Environ. Sci. 10, 1911-1916 (2017).

200. Leš, K. \& Jordan, C.-S. Ionic conductivity enhancement in solid polymer electrolytes by electrochemical in situ formation of an interpenetrating network. RSC Adv. 10, 41296-41304 (2020).

201. Dey, A., Karan, S. \& De, S. K. Effect of nanofillers on thermal and transport properties of potassium iodide-polyethylene oxide solid polymer electrolyte. Solid State Commun. 149, 1282-1287 (2009).

202. Waqas, M. et al. High-performance PE-BN/PVDF-HFP bilayer separator for lithium-ion batteries. Adv. Mater. Interfaces 6, 1-10 (2019).

203. Liu, W., Ullah, B., Kuo, C.-C. \& Cai, X. Two-dimensional nanomaterials-based polymer composites: fabrication and energy storage applications. Adv. Polym. Technol. 2019, 1-15 (2019).

204. Loste, J., Lopez-Cuesta, J. M., Billon, L., Garay, H. \& Save, M. Transparent polymer nanocomposites: an overview on their synthesis and advanced properties. Prog. Polym. Sci. 89, 133-158 (2019).

205. Wang, J. et al. Effective preparation of one-dimensional boron-nitridenanotube-supported nanosheet hierarchical structures and their optical/ adsorption properties. ChemistrySelect 3, 10832-10836 (2018)

206. Jiang, H. X. \& Lin, J. Y. Review-hexagonal boron nitride epilayers: growth, optical properties and device applications. ECS J. Solid State Sci. Technol. 6, Q3012-Q3021 (2017). 
207. Pakdel, A. et al. Facile synthesis of vertically aligned hexagonal boron nitride nanosheets hybridized with graphitic domains. J. Mater. Chem. 22, 4818-4824 (2012).

208. Hua, Li,L. et al. Photoluminescence of boron nitride nanosheets exfoliated by ball milling. Appl. Phys. Lett. 100, 261108 (2012).

209. Zhong, B. et al. Hollow BN microspheres constructed by nanoplates: Synthesis, growth mechanism and cathodoluminescence property. CrystEngComm 13, 819-826 (2011).

210. Liu, H. et al. High-performance deep ultraviolet photodetectors based on fewlayer hexagonal boron nitride. Nanoscale 10, 5559-5565 (2018).

211. Bisht, A., Kumar, V., Maity, P. C., Lahiri, I. \& Lahiri, D. Strong and transparent PMMA sheet reinforced with amine functionalized BN nanoflakes for UVshielding application. Compos. Part B Eng. 176, 107274 (2019).

212. Goodwin, D. G. et al. Graphene/polymer nanocomposite degradation by ultraviolet light: the effects of graphene nanofillers and their potential for release. Polym. Degrad. Stab. 182, 109365 (2020).

213. Zeranska-Chudek, K. et al. Study of the absorption coefficient of graphenepolymer composites. Sci. Rep. 8, 1-8 (2018).

214. Kuang, Z. et al. Fabrication of highly oriented hexagonal boron nitride nanosheet/elastomer nanocomposites with high thermal conductivity. Small 11, 1655-1659 (2015).

215. Wang, Q. H., Kalantar-Zadeh, K., Kis, A., Coleman, J. N. \& Strano, M. S. Electronics and optoelectronics of two-dimensional transition metal dichalcogenides. Nat Nanotechnol. 7, 699-712 (2012).

216. Liu, T. X. \& Huang, S. Properties and Applications of Polymer Nanocomposites Physical Properties and Applications of Polymer Nanocomposites (Springer Berlin Heidelberg, 2017). https://doi.org/10.1007/978-3-662-53517-2.

217. Liu, D. et al. Multifunctional polymer/porous boron nitride nanosheet membranes for superior trapping emulsified oils and organic molecules. Adv. Mater. Interfaces 2, 1500228 (2015).

218. Veličković, S. Application of nanocomposites in the automotive industry. Conference: International Congress Motor Vehicles \& Motors 323-332 (2018)

219. Rojaee, R. et al. Highly-cyclable room-temperature phosphorene polymer electrolyte composites for Li metal batteries. Adv. Funct. Mater. 30, 1910749 (2020).

220. Rasul, M. G., Anwar, T. Bin, Zaman, S., Bubli, S. Y. \& Kabir, K. B. Characterization of Local Biomass and Municipal Solid Waste To Assess the Potential As Fuels and Chemicals. in International Conference on Chemical Engineering 265-273 (2017).

221. Rodrigues, M. T. F. et al. Hexagonal boron nitride-based electrolyte composite for li-ion battery operation from room temperature to $150^{\circ} \mathrm{C}$. Adv. Energy Mater. 6, 1-7 (2016).

222. Luo, W. et al. A thermally conductive separator for stable Li metal anodes. Nano Lett. 15, 6149-6154 (2015).

223. Maksud, $M$. et al. Tunable nanomechanical performance regimes in ceramic nanowires. Nanotechnology 30, 47 (2019)

224. El Khalifi, M. et al. Encapsulation capacity and natural payload delivery of an anticancer drug from boron nitride nanotube. Phys. Chem. Chem. Phys. 18, 24994-25001 (2016).

225. Rasul, M. G., Ahmed, I. \& Hossain, M. I. Safe drinking water: effect of granular activated carbon bed characteristics on iron removal from water. Chem. Eng. Res. Bull. 18, 1-5 (2015).

226. Wang, C., Jagirdar, P., Naserifar, S. \& Sahimi, M. Molecular simulation study of gas solubility and diffusion in a polymer-boron nitride nanotube composite. J. Phys. Chem. B 120, 1273-1284 (2016)

227. Ganji, M. D., Sharifi, N., Fereidoon, A. \& Ahangari, M. G. Epoxy monomer adsorption on Group III (B, Al, Ga) nitride nanotubes: vdW-DF studies on mechanical and electronic properties. Superlattices Microstruct. 67, 127-143 (2014).

228. Yuan, F. et al. Scalable exfoliation for large-size boron nitride nanosheets by low temperature thermal expansion-assisted ultrasonic exfoliation. J. Mater. Chem. C. 5, 6359-6368 (2017).

229. Lei, W. et al. Boron nitride colloidal solutions, ultralight aerogels and freestanding membranes through one-step exfoliation and functionalization. Nat Commun. 6, 1-8 (2015).

230. Sainsbury, T. et al. Dibromocarbene functionalization of boron nitride nanosheets: toward band gap manipulation and nanocomposite applications. Chem. Mater. 26, 7039-7050 (2014)

231. Yang, N., Zeng, X., Lu, J., Sun, R. \& Wong, C. P. Effect of chemical functionalization on the thermal conductivity of 2D hexagonal boron nitride. Appl. Phys. Lett. 113, $171904 \backslash(2018)$.

232. Nazarov, A. S. et al. Functionalization and dispersion of hexagonal boron nitride (h-BN) nanosheets treated with inorganic reagents. Chem. - Asian J. 7, 554-560 (2012).

233. Sainsbury, T. et al. Oxygen radical functionalization of boron nitride nanosheets. J. Am. Chem. Soc. 134, 18758-18771 (2012)

234. Pakdel, A., Bando, Y. \& Golberg, D. Plasma-assisted interface engineering of boron nitride nanostructure films. ACS Nano 8, 10631-10639 (2014).
235. Hong, J. P. et al. Polyethylene/boron-containing composites for radiation shielding. Thermochim. Acta 585, 5-9 (2014)

236. Lee, D. et al. Scalable exfoliation process for highly soluble boron nitride nanoplatelets by hydroxide-assisted ball milling. Nano Lett. 15, 1238-1244 (2015).

237. Lin, Y., Williams, T. V. \& Connell, J. W. Soluble, exfoliated hexagonal boron nitride nanosheets. J. Phys. Chem. Lett. 1, 277-283 (2010).

238. Wang, H. M. et al. Stability and properties of the two-dimensional hexagonal boron nitride monolayer functionalized by hydroxyl $(\mathrm{OH})$ radicals: a theoretical study. J. Mol. Model. 19, 5143-5152 (2013).

239. Chen, M. S., Brandow, S. L., Schull, T. L., Chrisey, D. B. \& Dressick, W. J. A noncovalent approach for depositing spatially selective materials on surfaces. Adv. Funct. Mater. 15, 1364-1375 (2005).

240. Zeng, X. et al. Artificial nacre-like papers based on noncovalent functionalized boron nitride nanosheets with excellent mechanical and thermally conductive properties. Nanoscale 7, 6774-6781 (2015).

241. Lee, D. et al. Enhanced mechanical properties of epoxy nanocomposites by mixing noncovalently functionalized boron nitride nanoflakes. Small $\mathbf{9}$, 2602-2610 (2013).

242. He, Y. M., Wang, Q. Q., Liu, W. \& Liu, Y. S. Functionalization of boron nitride nanoparticles and their utilization in epoxy composites with enhanced thermal conductivity. Phys. Status Solidi Appl. Mater. Sci. 211, 677-684 (2014).

243. Cui, M. et al. Non-covalent functionalized hexagonal boron nitride nanoplatelets to improve corrosion and wear resistance of epoxy coatings. RSC Adv. 7 44043-44053 (2017)

244. Ma, P. \& Spencer, J. T. Non-covalent stabilization and functionalization of boron nitride nanosheets (BNNSs) by organic polymers: formation of complex BNNSscontaining structures. J. Mater. Sci. 50, 313-323 (2015).

245. Saggar, R., Porwal, H., Tatarko, P., Dlouhý, I. \& Reece, M. J. Boron nitride nanosheets reinforced glass matrix composites. Adv. Appl. Ceram. 114, S26-S33 (2015).

246. Jan, R., Sadiq, M. \& Hussain, A. Boron nitride-polymer composites: mechanical properties evaluation at various strain ratios. in 2017 14th International Bhurban Conference on Applied Sciences and Technology (IBCAST) 25-28 (IEEE, 2017).

247. Sun, Z. et al. High-purity production of ultrathin boron nitride nanosheets via shock chilling and their enhanced mechanical performance and transparency in nanocomposite hydrogels. Nanotechnology 29, 215602 (2018).

248. Wang, J. et al. Super-compatible functional boron nitride nanosheets/polymer films with excellent mechanical properties and ultra-high thermal conductivity for thermal management. J. Mater. Chem. C. 6, 1363-1369 (2018).

249. Vijayaraghavan, V. \& Zhang, L. Tensile and interfacial loading characteristics of boron nitride-carbon nanosheet reinforced polymer nanocomposites. Polym (Basel). 11, 1075 (2019).

250. Nikfar, N., Zare, Y. \& Rhee, K. Y. Dependence of mechanical performances of polymer/carbon nanotubes nanocomposites on percolation threshold. Phys. $B$ Condens. Matter 533, 69-75 (2018).

251. Zare, Y. \& Rhee, K. Y. Dependence of Z parameter for tensile strength of multilayered interphase in polymer nanocomposites to material and interphase properties. Nanoscale Res. Lett. 12, 42 (2017)

252. Zare, Y. \& Rhee, K. Y. Evaluation of the tensile strength in carbon nanotubereinforced nanocomposites using the expanded takayanagi model. JOM 71, 3980-3988 (2019)

253. Shen, X., Zheng, Q. \& Kim, J. Rational design of two-dimensional nanofillers for polymer nanocomposites toward multifunctional applications. Prog. Mater. Sci. 115, 100708 (2021).

254. Joy, J., George, E., Haritha, P., Thomas, S. \& Anas, S. An overview of boron nitride based polymer nanocomposites. J. Polym. Sci. 58, 3115-3141 (2020).

255. Wang, J., Ma, F. \& Sun, M. Graphene, hexagonal boron nitride, and their heterostructures: properties and applications. RSC Adv. 7, 16801-16822 (2017).

256. Zhang, K., Feng, Y., Wang, F., Yang, Z. \& Wang, J. Two dimensional hexagonal boron nitride (2D-hBN): Synthesis, properties and applications. J. Mater. Chem. C. 5, 11992-12022 (2017).

257. Bonaccorso, F. et al. Graphene, related two-dimensional crystals, and hybrid systems for energy conversion and storage. Science 347, 1246501-9 (2015).

258. Belyaeva, L. A., van Deursen, P. M. G., Barbetsea, K. I. \& Schneider, G. F. Hydrophilicity of graphene in water through transparency to polar and dispersive interactions. Adv. Mater. 30, (2018).

259. Pelin, M. et al. Differential cytotoxic effects of graphene and graphene oxide on skin keratinocytes. Sci. Rep. 7, 1-12 (2017).

260. Feng, S. et al. Folate-conjugated boron nitride nanospheres for targeted delivery of anticancer drugs. Int. J. Nanomed. 11, 4573-4582 (2016).

261. Wei, X., Wang, M. S., Bando, Y. \& Golberg, D. Tensile tests on individual multiwalled boron nitride nanotubes. Adv. Mater. 22, 4895-4899 (2010).

262. Nan, H. Y. et al. The thermal stability of graphene in air investigated by Raman spectroscopy. J. Raman Spectrosc. 44, 1018-1021 (2013). 
263. Yoon, D., Son, Y. W. \& Cheong, H. Negative thermal expansion coefficient of graphene measured by raman spectroscopy. Nano Lett. 11, 3227-3231 (2011).

264. Mashreghi, A. Thermal expansion/contraction of boron nitride nanotubes in axial, radial and circumferential directions. Comput. Mater. Sci. 65, 356-364 (2012).

265. Sevik, C. Assessment on lattice thermal properties of two-dimensional honeycomb structures: graphene, h-BN, h -MoS 2, and h -MoSe 2. Phys. Rev. B Condens. Matter Mater. Phys. 89, 1-5 (2014).

266. Steinborn, C. et al. Correlation between microstructure and electrical resistivity of hexagonal boron nitride ceramics. J. Eur. Ceram. Soc. 33, 1225-1235 (2013)

267. Auwärter, W. Hexagonal boron nitride monolayers on metal supports: Versatile templates for atoms, molecules and nanostructures. Surf. Sci. Rep. 74, 1-95 (2019).

\section{ACKNOWLEDGEMENTS}

This project is financially supported by Ford Motor Company. The authors also express sincere gratitude to Mr. Md Ruhul Amin Shikder from the University of Illinois at Chicago for his altruism by reading the full manuscript, editing, and giving critical suggestions to make this manuscript suitable for publications.

\section{AUTHOR CONTRIBUTIONS}

R.S.Y. and B.A. directed and supervised the project. M.G.R. drafted the manuscript with inputs from A.K. All the authors contributed to the revision and discussion of the manuscript.

\section{COMPETING INTERESTS}

The authors declare no competing interests.

\section{ADDITIONAL INFORMATION}

Correspondence and requests for materials should be addressed to B.A. or R.S-Y.

Reprints and permission information is available at http://www.nature.com/ reprints

Publisher's note Springer Nature remains neutral with regard to jurisdictional claims in published maps and institutional affiliations.

(i) Open Access This article is licensed under a Creative Commons Attribution 4.0 International License, which permits use, sharing, adaptation, distribution and reproduction in any medium or format, as long as you give appropriate credit to the original author(s) and the source, provide a link to the Creative Commons license, and indicate if changes were made. The images or other third party material in this article are included in the article's Creative Commons license, unless indicated otherwise in a credit line to the material. If material is not included in the article's Creative Commons license and your intended use is not permitted by statutory regulation or exceeds the permitted use, you will need to obtain permission directly from the copyright holder. To view a copy of this license, visit http://creativecommons. org/licenses/by/4.0/.

(c) The Author(s) 2021 